\title{
Hadronization in semi-inclusive deep-inelastic scattering on nuclei
}

\section{HERMES Collaboration}

A. Airapetian ${ }^{\mathrm{p}}$, N. Akopov aa, Z. Akopov ${ }^{\text {aa }}$, E.C. Aschenauer ${ }^{\mathrm{g}}$, W. Augustyniak ${ }^{\mathrm{z}}$, R. Avakian ${ }^{\text {aa }}$, A. Avetissian ${ }^{\text {aa }}$, E. Avetissian ${ }^{\mathrm{k}}$, N. Bianchi ${ }^{\mathrm{k}}$, H.P. Blok ${ }^{\mathrm{r}, \mathrm{y}}$, H. Böttcher ${ }^{\mathrm{g}}$, C. Bonomo ${ }^{\mathrm{j}}$, A. Borissov ${ }^{\mathrm{n}}$, A. Brüll, V. Bryzgalov ${ }^{\mathrm{t}}$, M. Capiluppi ${ }^{\mathrm{j}}$, G.P. Capitani ${ }^{\mathrm{k}}$, E. Cisbani ${ }^{\mathrm{v}}$, G. Ciullo $^{j}$, M. Contalbrigo ${ }^{j}$, P.F. Dalpiaz ${ }^{j}$, W. Deconinck ${ }^{p}$, R. De Leo ${ }^{b}$, M. Demey ${ }^{\mathrm{r}}$, L. De Nardo ${ }^{\mathrm{f}}$, E. De Sanctis ${ }^{\mathrm{k}}$, M. Diefenthaler ${ }^{\mathrm{i}}$, P. Di Nezza ${ }^{\text {k }}$, J. Dreschler ${ }^{\mathrm{r}}$, M. Düren ${ }^{\mathrm{n}}$, M. Ehrenfried ${ }^{\mathrm{i}}$, G. Elbakian ${ }^{\text {aa }}$, F. Ellinghaus ${ }^{\text {e }}$, U. Elschenbroich ${ }^{1}$, R. Fabbri ${ }^{\mathrm{g}}$, A. Fantoni ${ }^{\mathrm{k}}, \mathrm{S}$. Frullani ${ }^{\mathrm{v}}$, D. Gabbert ${ }^{\text {g }}$, G. Gapienko ${ }^{\text {t }}$, V. Gapienko ${ }^{\text {, }}$, F. Garibaldi ${ }^{\mathrm{v}}$, G. Gavrilov ${ }^{\mathrm{f}, \mathrm{s}, \mathrm{w}}$, V. Gharibyan ${ }^{\text {aa }}$, F. Giordano ${ }^{j}$, S. Gliske ${ }^{\mathrm{p}}$, L. Grigoryan ${ }^{\text {aa }}$, C. Hadjidakis ${ }^{\text {k }}$, M. Hartig ${ }^{\text {f }}$, D. Hasch ${ }^{\text {k }}$, T. Hasegawa ${ }^{\mathrm{x}}$, G. Hill ${ }^{\mathrm{n}}$, A. Hillenbrand ${ }^{\mathrm{i}}$, M. Hoek ${ }^{n}$, B. Hommez ${ }^{1}$, I. Hristova ${ }^{g}$, Y. Imazu ${ }^{\mathrm{x}}$, A. Ivanilov ${ }^{\mathrm{t}}$, H.E. Jackson ${ }^{\text {a }, ~ R . ~ K a i s e r ~}{ }^{\text {n }}$, T. Keri ${ }^{n}$, E. Kinney ${ }^{\mathrm{e}}$, A. Kisselev ${ }^{\mathrm{o}, \mathrm{s}}$, M. Kopytin ${ }^{\mathrm{g}}$, V. Korotkov ${ }^{\mathrm{t}}$, P. Kravchenko ${ }^{\mathrm{s}}$, L. Lagamba ${ }^{\mathrm{b}}, \mathrm{K}_{\text {R. Lamb }}{ }^{\text {, }}$, L. Lapikás ${ }^{r}$, I. Lehmann ${ }^{n}$, P. Lenisa ${ }^{j}$, P. Liebing ${ }^{g}$, L.A. Linden-Levy ${ }^{\circ}$, W. Lorenzon ${ }^{p}$, S. Lu ${ }^{n}$, X. Lu ${ }^{x}$, B. Maiheu ${ }^{1}$, N.C.R. Makins ${ }^{\circ}$, B. Marianski ${ }^{\mathrm{z}}$, H. Marukyan ${ }^{\text {aa }}$, V. Mexner $^{\mathrm{r}}$, C.A. Miller ${ }^{\mathrm{w}}$, Y. Miyachi ${ }^{\mathrm{x}}$, V. Muccifora ${ }^{\mathrm{k}}$, M. Murray ${ }^{\mathrm{n}}$, A. Mussgiller ${ }^{\mathrm{i}}$, E. Nappi $^{\mathrm{b}}$, Y. Naryshkin ${ }^{\mathrm{s}}$, A. Nass ${ }^{i}$, M. Negodaev ${ }^{g}$, W.-D. Nowak ${ }^{g}$, L.L. Pappalardo ${ }^{j}$, R. Perez-Benito ${ }^{\text {}}$, N. Pickert ${ }^{\mathrm{i}}$, M. Raithel ${ }^{\mathrm{i}}$, D. Reggiani ${ }^{\mathrm{i}}$, P.E. Reimer ${ }^{\mathrm{a}}$, A. Reischl ${ }^{\mathrm{r}}$, A.R. Reolon ${ }^{\mathrm{k}}$, C. Riedl ${ }^{\mathrm{g}}$, K. Rith ${ }^{\mathrm{i}}$, S.E. Rock ${ }^{\mathrm{f}}$, G. Rosner ${ }^{\mathrm{n}}$, A. Rostomyan ${ }^{\text {f }}$, L. Rubacek ${ }^{\mathrm{n}}$, J. Rubin ${ }^{\circ}$, D. Ryckbosch ${ }^{1}$, Y. Salomatin ${ }^{\mathrm{t}}$, A. Schäfer ${ }^{u}$, G. Schnell ${ }^{x}$, B. Seitz ${ }^{n}$, C. Shearer ${ }^{n}$, T.-A. Shibata ${ }^{\mathrm{x}}$, V. Shutov ${ }^{\text {h }}$, M. Stancari ${ }^{j}$, M. Statera ${ }^{j}$, J.J.M. Steijger ${ }^{r}$, H. Stenzel ${ }^{\mathrm{n}}$, J. Stewart $^{\mathrm{g}, *}$, F. Stinzing ${ }^{\mathrm{i}}$, J. Streit ${ }^{\mathrm{n}}$, S. Taroian ${ }^{\text {aa }}$, B. Tchuiko ${ }^{\mathrm{t}}$, 


\title{
A. Trzcinski ${ }^{z}$, M. Tytgat ${ }^{1}$, A. Vandenbroucke ${ }^{1}$, P.B. van der Nat ${ }^{r}$, G. van der Steenhoven ${ }^{r}$, Y. van Haarlem ${ }^{1}$, C. van Hulse ${ }^{1}$, M. Varanda ${ }^{\mathrm{f}}$, D. Veretennikov ${ }^{\mathrm{s}}$, V. Vikhrov ${ }^{\mathrm{s}}$, I. Vilardi ${ }^{\mathrm{b}}$, C. Vogel $^{\mathrm{i}}, \mathrm{S}$. Wang ${ }^{\mathrm{c}}$, S. Yaschenko ${ }^{i}, Y . Y^{d}, Z$ Z. Ye ${ }^{\text {f }}$, S. Yen ${ }^{w}$, W. Yu ${ }^{n}$, D. Zeiler ${ }^{i}$, B. Zihlmann ${ }^{1}$, P. Zupranski ${ }^{\mathrm{z}}$
}

a Physics Division, Argonne National Laboratory, Argonne, IL 60439-4843, USA

${ }^{\mathrm{b}}$ Istituto Nazionale di Fisica Nucleare, Sezione di Bari, 70124 Bari, Italy

c School of Physics, Peking University, Beijing 100871, China

d Department of Modern Physics, University of Science and Technology of China, Hefei, Anhui 230026, China

e Nuclear Physics Laboratory, University of Colorado, Boulder, CO 80309-0390, USA

${ }^{\mathrm{f}}$ DESY, 22603 Hamburg, Germany

g DESY, 15738 Zeuthen, Germany

h Joint Institute for Nuclear Research, 141980 Dubna, Russia

i Physikalisches Institut, Universität Erlangen-Nürnberg, 91058 Erlangen, Germany

j Istituto Nazionale di Fisica Nucleare, Sezione di Ferrara and Dipartimento di Fisica, Università di Ferrara, 44100 Ferrara, Italy

${ }^{\mathrm{k}}$ Istituto Nazionale di Fisica Nucleare, Laboratori Nazionali di Frascati, 00044 Frascati, Italy

${ }^{1}$ Department of Subatomic and Radiation Physics, University of Gent, 9000 Gent, Belgium

$\mathrm{m}$ Physikalisches Institut, Universität Gießen, 35392 Gießen, Germany

${ }^{\mathrm{n}}$ Department of Physics and Astronomy, University of Glasgow, Glasgow G12 8QQ, United Kingdom

o Department of Physics, University of Illinois, Urbana, IL 61801-3080, USA

p Randall Laboratory of Physics, University of Michigan, Ann Arbor, MI 48109-1040, USA

q Lebedev Physical Institute, 117924 Moscow, Russia

${ }^{\mathrm{r}}$ Nationaal Instituut voor Kernfysica en Hoge-Energiefysica (NIKHEF), 1009 DB Amsterdam, The Netherlands

${ }^{\mathrm{s}}$ Petersburg Nuclear Physics Institute, St. Petersburg, Gatchina 188350, Russia

${ }^{\mathrm{t}}$ Institute for High Energy Physics, Protvino, Moscow region 142281, Russia

u Institut für Theoretische Physik, Universität Regensburg, 93040 Regensburg, Germany

v Istituto Nazionale di Fisica Nucleare, Sezione Roma 1, Gruppo Sanità and Physics Laboratory, Istituto Superiore di Sanità, 00161 Roma, Italy

${ }^{\mathrm{w}}$ TRIUMF, Vancouver, British Columbia V6T 2A3, Canada

${ }^{\mathrm{x}}$ Department of Physics, Tokyo Institute of Technology, Tokyo 152, Japan

${ }^{\mathrm{y}}$ Department of Physics and Astronomy, Vrije Universiteit, $1081 \mathrm{HV}$ Amsterdam, The Netherlands

${ }^{\mathrm{z}}$ Andrzej Soltan Institute for Nuclear Studies, 00-689 Warsaw, Poland

aa Yerevan Physics Institute, 375036 Yerevan, Armenia

Received 26 April 2007; received in revised form 5 June 2007; accepted 8 June 2007

Available online 15 June 2007

\begin{abstract}
A series of semi-inclusive deep-inelastic scattering measurements on deuterium, helium, neon, krypton, and xenon targets has been performed in order to study hadronization. The data were collected with the HERMES detector at the DESY laboratory using a $27.6 \mathrm{GeV}$ positron or electron beam. Hadron multiplicities on nucleus $A$ relative to those on the deuteron, $R_{A}^{h}$, are presented for various hadrons $\left(\pi^{+}, \pi^{-}, \pi^{0}\right.$, $K^{+}, K^{-}, p$, and $\bar{p}$ ) as a function of the virtual-photon energy $v$, the fraction $z$ of this energy transferred to the hadron, the photon virtuality $Q^{2}$, and the hadron transverse momentum squared $p_{t}^{2}$. The data reveal
\end{abstract}

\footnotetext{
* Corresponding author.

E-mail address: james.stewart@desy.de (J. Stewart).
} 
a systematic decrease of $R_{A}^{h}$ with the mass number $A$ for each hadron type $h$. Furthermore, $R_{A}^{h}$ increases (decreases) with increasing values of $v(z)$, increases slightly with increasing $Q^{2}$, and is almost independent of $p_{t}^{2}$, except at large values of $p_{t}^{2}$. For pions two-dimensional distributions also are presented. These indicate that the dependences of $R_{A}^{\pi}$ on $v$ and $z$ can largely be described as a dependence on a single variable $L_{c}$, which is a combination of $v$ and $z$. The dependence on $L_{c}$ suggests in which kinematic conditions partonic and hadronic mechanisms may be dominant. The behaviour of $R_{A}^{\pi}$ at large $p_{t}^{2}$ constitutes tentative evidence for a partonic energy-loss mechanism. The $A$-dependence of $R_{A}^{h}$ is investigated as a function of $\nu, z$, and of $L_{c}$. It approximately follows an $A^{\alpha}$ form with $\alpha \approx 0.5-0.6$.

(C) 2007 Elsevier B.V. All rights reserved.

Keywords: Nuclei; Quarks; Hadron production; Hadronization; Attenuation; A-dependence

\section{Introduction}

After decades of extensive study, understanding the confinement of quarks and gluons in hadrons still is one of the great challenges in hadronic physics. To uncover its nature, hadronic reactions in a nuclear medium, either cold or hot, are studied. Typical examples are the measurements of hadron production on nuclear targets in semi-inclusive deep-inelastic scattering of leptons [1-5] and the jet-quenching and parton energy-loss phenomena observed in ultrarelativistic heavy-ion collisions [6,7]. In each case hadron yields are observed that are different from those observed in the corresponding reactions on free nucleons.

The process that leads from the partons produced in the elementary interaction to the hadrons observed experimentally is commonly referred to as hadronization or fragmentation. According to theoretical estimates the hadronization process occurs over length scales varying from less than a femtometer to several tens of femtometers. At these length scales the magnitude of the strong coupling constant is such that perturbative techniques cannot be applied. Hence, hadronization is an intrinsically non-perturbative QCD process, for which only approximate theoretical approaches are presently available. Experimental data are vital for supporting these theoretical developments, since they can be used to gauge or guide the calculations.

The hadronization process in free space has been studied extensively in $e^{+} e^{-}$annihilation experiments [8]. As a result the spectra of particles produced and their kinematic dependences are rather well known. However, little is known about the space-time evolution of the process. Semi-inclusive production of hadrons in deep-inelastic scattering of leptons from atomic nuclei provides a way to investigate this space-time development. Leptoproduction of hadrons has the virtue that the energy and the momentum of the struck parton are well determined, as they are tagged by the scattered lepton. By using nuclei of increasing size one can investigate the time development of hadronization. If hadronization occurs quickly, i.e., if the hadrons are produced at small distances compared to the size of atomic nuclei, the relevant interactions in the nuclear environment involve well-known hadronic cross sections such as the ones for pion-nucleon interactions. If, in contrast, hadronization occurs over large distances, the relevant interactions are partonic and involve the emission of gluons and quark-antiquark pairs. The two mechanisms lead to different predictions for the decrease in hadron yield, known as attenuation, on nuclei as compared to that on free nucleons.

Most likely, a combination of these two mechanisms contributes to the observed attenuation of hadron yields on nuclei. This expectation has led to a range of phenomenological approaches, which are briefly summarized in the next section. The available calculations cover a range of possible mechanisms (partonic energy loss or hadronic absorption) and time (length) scales (from 
less than $1 \mathrm{fm}$ to more than $10 \mathrm{fm}$ ), which results in different dependences on the various kinematic variables. In order to distinguish between these calculations precise hadron attenuation data are needed as a function of several kinematic variables for a range of nuclei and for several hadron types.

Exploratory measurements were first performed at SLAC [1] and later by the EMC [2] and E665 [3] Collaborations. More recently many more data have been collected by the HERMES Collaboration at DESY and the CLAS Collaboration at the Thomas Jefferson National Accelerator Facility [9]. Some of the HERMES data have been published already [4,5]. The CLAS data are presently being analysed [10]. In this paper we present the full results from HERMES on the multiplicities for the production of pions, kaons, protons, and antiprotons on helium, neon, krypton, and xenon targets relative to those for deuterium. It goes beyond the scope of the present paper to compare the data to the available theoretical calculations. Instead, prominent features of the data are identified and used to address two key issues in the study of hadronization: (i) what are the time or length scales of the process, and (ii) what are the mechanisms that lead to nuclear attenuation?

The paper is organized as follows. In Section 2 the theoretical framework is described, and some representative theoretical models are summarized. In Section 3 those aspects of the HERMES experiment that are relevant to the present measurements are presented. In Section 4 the data analysis is discussed, including the corrections to the raw data and the systematic uncertainties. The results for the attenuation as a function of various kinematic variables are presented and discussed in Section 5. This section has several subsections in which the features of the data, especially those related to the relevant time scales and mechanisms, and the $A$-dependence, are discussed separately. The results are summarized in the last section, which also lists the conclusions.

\section{Theoretical framework}

In order to put the experimental results into perspective, in this section the concepts that are used in the study of hadronization are presented, and models that have been developed to describe the experimental results are briefly discussed. It is emphasized that the latter is meant only to illustrate potential interpretations of the data. The experimental data and the features that they exhibit are the genuine subject.

The hadronization process in a nuclear medium can be studied by means of semi-inclusive deep-inelastic scattering (SIDIS) of electrons or positrons from nuclei. For that purpose the multiplicity ratio $R_{A}^{h}$ is introduced, which is defined as the ratio of the number of hadrons $h$ produced per deep-inelastic scattering (DIS) event on a nuclear target with mass number $A$ to that for a deuterium (D) target. Fig. 1 illustrates the definition of the relevant lepton and hadron kinematic variables. The ratio $R_{A}^{h}$ depends on the leptonic variables $v$, the energy of the virtual photon, and $Q^{2}$, the negative of the four-momentum of the virtual photon squared, and on the hadronic variables $z=E_{h} / v$, the fraction of the virtual-photon energy carried by the hadron, and $p_{t}^{2}$, the square of the hadron momentum component transverse to the direction of the virtual photon. Thus $R_{A}^{h}$ can be written as:

$$
R_{A}^{h}\left(v, Q^{2}, z, p_{t}^{2}\right)=\frac{\left(\frac{N^{h}\left(v, Q^{2}, z, p_{t}^{2}\right)}{N^{e}\left(v, Q^{2}\right)}\right)_{A}}{\left(\frac{N^{h}\left(v, Q^{2}, z, p_{t}^{2}\right)}{N^{e}\left(v, Q^{2}\right)}\right)_{D}}
$$




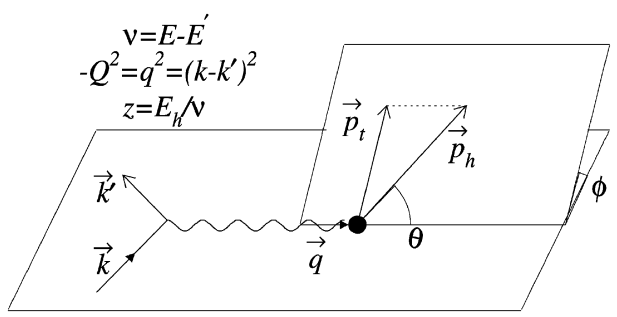

Fig. 1. Kinematic planes for hadron production in semi-inclusive deep-inelastic scattering and definitions of the relevant lepton and hadron variables. The quantities $k=(E, \vec{k})$ and $k^{\prime}=\left(E^{\prime}, \vec{k}^{\prime}\right)$ are the four-momenta of the incident and scattered lepton, $\left(E_{h}, \vec{p}_{h}\right)$ is the four-momentum of the produced hadron, and $\vec{p}_{t}$ is the transverse momentum of the hadron.

with $N^{h}\left(\nu, Q^{2}, z, p_{t}^{2}\right)$ the number of semi-inclusive hadrons at given $\left(\nu, Q^{2}, z, p_{t}^{2}\right)$, and $N^{e}\left(v, Q^{2}\right)$ the number of inclusive DIS leptons at $\left(v, Q^{2}\right)$. Implicit in this definition is the integration over the angle $\phi$ between the lepton scattering plane and the hadron production plane (see Fig. 1).

Experiments at large values of $v[2,3]$ give values $R_{A}^{h} \approx 1.0$ within the experimental uncertainty. This is interpreted as an indication that nuclear effects are negligible in that region. At lower values of $v$ the value of $R_{A}^{h}$ has been found to be well below unity $[1,4,5]$.

Even though hadronization is not yet quantitatively understood, it is generally assumed that the following processes play a role in leptoproduction of hadrons.

After a quark in a nucleon has absorbed the virtual photon, it can lose energy by scattering from other quarks and by radiating gluons. As a result the value of $R_{A}^{h}$ may be influenced. A change in $R_{A}^{h}$ can also result from the quark in a nucleus having a different distribution function as a result of partonic rescaling. These two effects will be called partonic mechanisms.

After a certain time a colourless object, called a prehadron, is formed, which has the quantum numbers of the final hadron, but not yet its full wave function. The concept of colour transparency [11] is closely related to this. The prehadron then evolves over some time into the physical hadron. In the Lund model [12] the process of (pre)hadron formation is described as the building of a colour field (string) between the struck quark and the residual system. The string gets stretched and breaks into smaller pieces, each with an (anti-)quark at both ends. The Lund model gives predictions (see, e.g., Ref. [13]) for the time $t_{c}$, and the corresponding (constituent or formation) length $l_{c}$, that it takes for the prehadron to be formed, and the time $t_{h}$ required for the final physical hadron to be formed. ${ }^{1}$

The average value of the formation length $l_{c}$, denoted by $L_{c}$, is given in the Lund model by

$$
L_{c}=f(z) \frac{v}{\kappa},
$$

where $f(z)$ is a function that goes as $1-z$ for large values of $z$ (this behaviour is also found in other models, and is due to the fact that in the limit $z \rightarrow 1$ the struck quark cannot have lost any energy when producing the final hadron), and has a broad maximum of about 0.4 around $z=0.35$. The quantity $\kappa$ is the string tension, which reflects the energy loss of the leading quark per unit length, usually taken as $\kappa=1 \mathrm{GeV} / \mathrm{fm}$. Thus, at values of $v$ of $5-25 \mathrm{GeV}$, the prehadron

\footnotetext{
1 In the literature the name 'formation time or length' has sometimes different meanings and also different symbols are used. Here the name 'formation length' $\left(l_{c}\right)$ is used for what is often called the prehadron (or constituent) formation length, and 'hadron formation length' $\left(l_{h}\right)$ for the hadron (or yo-yo) formation length (see, e.g., Refs. $\left.[13,14]\right)$.
} 
formation takes place over distances of about 1-10 fm, comparable to the size of a nucleus. The values of $l_{h}$ are larger than $l_{c}$, so the final hadron is often formed outside the nucleus.

If the (pre)hadron is formed inside the nucleus, it can experience hadronic interactions, generally called final-state interactions (FSI). For discussing the effects of these, we will not discriminate between prehadrons and hadrons, and just talk about hadrons and hadronic effects, although the relevant cross sections may be different for hadrons and prehadrons. The first effect of FSI may be the rescattering of the hadron from the nucleons in the nucleus, the hadron losing energy and possibly 'generating' other, mainly low energy, hadrons (if these are mesons, the word generate can be taken literally; if these are nucleons, generated means that they are now in a continuum state). The effect is thus a loss of hadrons at a given value of $z$, and an increase of the same hadron, but possibly also of other hadrons, at lower $z$. Another effect of FSI can be that the original hadron is absorbed. This is usually accompanied by the emission of other, again mainly low-energy, hadrons. So also in this case there is a loss of hadrons at given $z$, and an increase of other hadrons at lower $z$. For a full description of all these effects, coupled-channel calculations should be performed. However, since these are rather complicated, in models usually only absorption is considered. Clearly this neglects the generation of hadrons at lower values of $z$.

The change from partonic mechanisms to hadronic mechanisms, which depends on the formation length relative to the size of the nucleus, influences $R_{A}^{h}$. Thus the use of various nuclear targets allows one to investigate the space-time development of the hadronization process.

At present, reliable QCD calculations of quark hadronization (fragmentation) cannot yet be performed because of the major role of 'soft', i.e., non-perturbative processes. For that reason various types of models have been developed.

Phenomenological models [15-18] use formation times/lengths and absorption cross sections for the various hadrons in the nuclear medium. Various formulae for the formation lengths have been used, and in the more advanced versions two length scales, $l_{c}$ and $l_{h}$, are distinguished, as well as different absorption cross sections for the quark, the prehadron, and the final hadron. The absorption cross sections are usually adjusted to obtain the best description of the $v$ - and $z$-dependence of the experimental data.

Other (QCD-inspired) models focus on the energy loss that the struck quark experiences in the nuclear environment [19-22]. In Refs. [20,21] twist-4 contributions to the fragmentation functions resulting from multiple scattering and gluon bremsstrahlung in a nuclear medium are calculated. A nuclear attenuation proportional to $A^{2 / 3}$ is predicted, where the power $2 / 3$ results from the coherence of the gluon radiation process [23], which gives an induced radiative energy loss of a quark traversing a length $L$ of matter proportional to $L^{2}$ [24]. No hadron absorption is included, as it is assumed that the hadron is formed outside the nucleus.

The effect of a finite formation length is included in Ref. [22], in which fragmentation functions are calculated that are again modified due to partonic energy loss in the nuclear medium during the time $t_{c}$. By using the quark energy loss determined from Drell-Yan data, a reasonable agreement with existing data is found. In order to keep the approach as simple as possible, absorption of the produced (pre)hadron is not taken into account.

Another class of models includes (pre)hadron absorption, with or without a description of what happens in the hadronization process. In Ref. [25] $R_{A}^{h}$ is described in terms of mediummodified fragmentation functions supplemented by nuclear absorption. A parton-rescaling model that has also been used to describe the EMC effect is used to describe the nuclear modification of the fragmentation functions. The (average) formation length is taken from the Lund model [12]. Ref. [26] calculates the nuclear attenuation of the (leading) hadron with $z>0.5$ by including as major ingredients the formation length $l_{c}$ and an absorption cross section of the prehadron. 
The effect of quark energy loss is found to be small. In Ref. [27] the hadron attenuation is investigated within the framework of the Boltzmann-Uehling-Uhlenbeck transport model. Since this is a coupled-channel approach, hadrons are not only absorbed, but can also be produced. Some choices for the formation time $t_{c}$, including taking it to be zero, are studied.

The theoretical calculations have been compared to the data from Refs. [2,4,5]. Notwithstanding their different and sometimes orthogonal ingredients, all models reproduce the global features of the data. In the case of the $v$-dependence, which is best described, the reason may be that the decreasing attenuation with increasing value of $v$ is largely due to a simple increase of the formation time $t_{c}$ with $v$ in the target rest frame due to Lorentz dilatation. The dependence of $R_{A}^{h}$ on $z$ and $A$, which in general is less well described, may be more discriminating, especially when more detailed data are available.

\section{Experiment}

The measurements were performed with the HERMES spectrometer [28] using a $27.6 \mathrm{GeV}$ positron or electron beam stored in the HERA ring at DESY. Some data were collected using a $12.0 \mathrm{GeV}$ positron beam [29,30], but since the amount of data was much less and only pions or all hadrons were identified, they are not included here. Typical beam currents were $40 \mathrm{~mA}$ down to $5 \mathrm{~mA}$. The spectrometer consists of two mirror-symmetric halves, located above and below the lepton beam pipe. A flux-exclusion plate in the midplane of the magnet prevents deflection of the lepton (and proton) beams passing through the center of the spectrometer. The scattered leptons and the produced hadrons were detected within an angular acceptance of $\pm 170 \mathrm{mrad}$ horizontally and \pm (40-140) mrad vertically. The lepton trigger was formed by a coincidence between signals from three scintillator hodoscope planes and a lead-glass calorimeter. A minimum energy deposit in the latter of $3.5 \mathrm{GeV}(1.4 \mathrm{GeV})$ for unpolarized (polarized) target runs was required.

The data were collected during the years 1999, 2000, 2004, and 2005, using unpolarized nuclear (He, $\mathrm{Ne}, \mathrm{Kr}, \mathrm{Xe}$ ) and polarized or unpolarized deuterium (D) gaseous targets internal to the storage ring (see Table 1). In 1997 also data on nitrogen were taken, but since at that time the RICH detector (see below) was not yet available, only data for pions and all hadrons together could be presented [4]. Therefore these data are not included here. The yields from polarized deuterium were summed over the two spin orientations. The target gases were injected into a $40 \mathrm{~cm}$ long tubular open-ended storage cell. Using an Unpolarized Gas Feed System [31] it is possible to provide $\mathrm{D}, \mathrm{He}, \mathrm{N}, \mathrm{Ne}, \mathrm{Kr}$, or Xe targets with relatively high areal densities (between $10^{14}$ and $10^{17}$ nucleons $/ \mathrm{cm}^{2}$ ), resulting in luminosities ranging from $10^{31}$ to $10^{33} \mathrm{~cm}^{-2} \mathrm{~s}^{-1}$. Such highdensity runs were taken at the end of HERA fills, with typical currents of 15 to $5 \mathrm{~mA}$ and beam lifetimes of one hour. This made it possible to accumulate the data for these targets in only a few days of integrated beam time. The luminosity was measured using elastic scattering of the

Table 1

Overview of the HERMES nuclear attenuation measurements

\begin{tabular}{lllll}
\hline Year & $E(\mathrm{GeV})$ & Target & Identified hadrons & Ref. \\
\hline 1997 & 27.6 & $\mathrm{D}, \mathrm{N}$ & $h^{ \pm}, \pi^{ \pm}$ & [4], [5] \\
1999 & 27.6 & $\mathrm{D}, \mathrm{Kr}$ & $h^{ \pm}, \pi^{ \pm}, \pi^{0}, K^{ \pm}, p, \bar{p}$ & [5], this work \\
2000 & 27.6 & $\mathrm{D}, \mathrm{He}, \mathrm{Ne}$ & $\pi^{ \pm}, \pi^{0}, K^{ \pm}, p, \bar{p}$ & this work \\
2000 & 12.0 & $\mathrm{D}, \mathrm{N}, \mathrm{Kr}$ & $h^{ \pm}, \pi^{ \pm}$ & {$[29]$} \\
2004 & 27.6 & $\mathrm{D}, \mathrm{Kr}, \mathrm{Xe}$ & $\pi^{ \pm}, K^{ \pm}, \pi^{0}, p, \bar{p}$ & this work \\
2005 & 27.6 & $\mathrm{D}, \mathrm{Kr}, \mathrm{Xe}$ & $\pi^{ \pm}, K^{ \pm}, \pi^{0}, p, \bar{p}$ & this work \\
\hline
\end{tabular}


beam particles from the electrons in the target gas: Bhabha scattering for a positron beam, Møller scattering for an electron beam [32]. There are several particle identification (PID) detectors in the HERMES spectrometer. Details on the performance and use of these PID detectors can be found in Ref. [33]. Electrons and positrons are identified by combining the information from a lead-glass calorimeter, a scintillator hodoscope preceded by two radiation lengths of lead (the pre-shower detector), and a transition-radiation detector.

The identification of charged pions, kaons, protons, and antiprotons is accomplished using the information from the Ring-Imaging Čerenkov detector (RICH) [34], which replaces a threshold Čerenkov counter used in the previously reported measurements on nitrogen [4]. The RICH detector uses two radiators, a $5 \mathrm{~cm}$ thick wall of silica-aerogel tiles followed by a large volume of $\mathrm{C}_{4} \mathrm{~F}_{10}$ gas, to provide separation of pions, kaons, and (anti)protons. Together these provide good particle identification for charged hadrons in the momentum range $2<p<15 \mathrm{GeV}$, with limited contamination from misidentified hadrons. The identification efficiencies and contaminations for pions, kaons, protons, and antiprotons were determined in a Monte Carlo simulation as a function of the hadron momentum and multiplicity in the relevant detector half. These performance parameters were verified in a limited kinematic domain using known particle species from identified resonance decays. They were used in a matrix method to unfold the true hadron distributions from the measured ones. Systematic uncertainties in the unfolding were estimated by using matrices determined in different ways, see Refs. [34,35] for details.

The electromagnetic calorimeter [36] provides neutral pion identification through the detection of two clusters without an accompanying track, originating from the two decay photons.

\section{Data analysis}

The analysis procedure is similar to the one described in detail in Refs. [4,5], where the nitrogen and first krypton data were presented. Since the publication of Ref. [5], more data on krypton were taken and all data were analysed in a wider kinematic range.

The hadron multiplicity ratio $R_{A}^{h}$ as defined in Eq. (1) was determined as a function of the leptonic $\left(Q^{2}\right.$ and $\left.v\right)$ and hadronic $\left(z\right.$ and $\left.p_{t}^{2}\right)$ variables for all identified particles and all targets. The kinematic constraints imposed on the scattered leptons were identical for all analysed data: $Q^{2}>1 \mathrm{GeV}^{2}, W=\sqrt{2 M v+M^{2}-Q^{2}}>2 \mathrm{GeV}$ (where $M$ is the nucleon mass) for the invariant mass of the photon-nucleon system, and $y=v / E<0.85$ for the energy fraction of the virtual photon. The constraints on $W$ and $y$ were applied in order to exclude nucleon resonances and to limit the magnitude of the radiative corrections to $R_{A}^{h}$, respectively. The resulting value of $x_{\mathrm{Bj}}=Q^{2} / 2 M v$ ranged from 0.023 to 0.8 .

As mentioned in the previous section, charged hadrons were identified in the momentum range 2.0-15.0 GeV by using the RICH detector. For the identification of the neutral pions through the detection of their decay photons, each of the two photon clusters was required to have an energy of at least $0.8 \mathrm{GeV}$. The background was evaluated in each kinematic bin by fitting the twophoton invariant mass spectrum with a Gaussian plus a polynomial that fits the shape of the background due to uncorrelated photons. The number of detected neutral pions was obtained by integrating the peak, corrected for background, over a $\pm 2 \sigma$ range with respect to the centroid of the Gaussian. The low momentum limit for the neutral pions was set at $2.5 \mathrm{GeV}$ in order to reduce backgrounds.

The integrated luminosities for all years and targets are listed in Table 2. Typical numbers of observed DIS leptons and identified hadrons are listed in Table 3. 
Table 2

Integrated luminosities (in $\mathrm{pb}^{-1}$ ) for the various data sets

\begin{tabular}{lllllr}
\hline Target & 1999 & 2000 & 2004 & 2005 & Sum \\
\hline $\mathrm{D}$ & 32.3 & 119.7 & 35.7 & 61.7 & 249.4 \\
$\mathrm{He}$ & & 27.9 & & & 27.9 \\
$\mathrm{Ne}$ & & 84.2 & 29.5 & 21.1 & 84.2 \\
$\mathrm{Kr}$ & 26.1 & 21.2 & 21.4 & 76.7 \\
$\mathrm{Xe}$ & & & & 42.6 \\
\hline
\end{tabular}

Table 3

Number of DIS leptons and identified hadrons collected on deuterium and krypton targets in 1999, 2004, and 2005 combined. The numbers are for the following kinematic constraints: $Q^{2}>1 \mathrm{GeV}^{2}, v>6 \mathrm{GeV}, W>2 \mathrm{GeV}$, and $z>0.2$

\begin{tabular}{llllrrrrr}
\hline Target & DIS & $\pi^{+}$ & $\pi^{-}$ & $\pi^{0}$ & $K^{+}$ & $K^{-}$ & \multicolumn{1}{l}{$p$} \\
\hline $\mathrm{D}$ & $6669 \mathrm{k}$ & $706 \mathrm{k}$ & $575 \mathrm{k}$ & $232 \mathrm{k}$ & $146 \mathrm{k}$ & $62 \mathrm{k}$ & $131 \mathrm{k}$ & $23 \mathrm{k}$ \\
$\mathrm{Kr}$ & $3516 \mathrm{k}$ & $286 \mathrm{k}$ & $232 \mathrm{k}$ & $90 \mathrm{k}$ & $68 \mathrm{k}$ & $26 \mathrm{k}$ & $69 \mathrm{k}$ & $8 \mathrm{k}$ \\
\hline
\end{tabular}

Most of the systematic uncertainties related to the detector, the reconstruction efficiencies and particle identification practically cancel in the ratio of the multiplicities. In determining the multiplicity ratios, deuterium data collected in the same year as the data for the heavier target were used to avoid uncertainties due to possible different conditions or functioning of the HERMES spectrometer during the years. It was verified that the multiplicity ratios obtained in different years were consistent within the statistical and systematic uncertainties.

The multiplicity ratios were also inspected as a function of the hadron angles $\theta_{x}$ and $\theta_{y}$ with respect to the beam direction in order to investigate whether the values of $R_{A}^{h}$ depend on the geometrical acceptance of the spectrometer. After applying (small) corrections for changes in average kinematics with $\theta_{x}$ or $\theta_{y}$, no effect was found beyond the statistical and systematic uncertainties. The dependence of the value of $R_{A}^{h}$ on the azimuthal angle $\phi$ of the hadron (see Fig. 1) was also investigated, since it is known that the SIDIS cross section on the proton and deuteron depends on this variable. It was found that $R_{A}^{h}$ was constant as a function of $\phi$ within the statistical and systematic uncertainties.

The data for the multiplicity ratios were corrected for radiative processes in the manner described in Ref. [37]. The code of Ref. [38] was modified to include the measured SIDIS cross sections. The radiative corrections (RC) were applied to both the inclusive and the semi-inclusive parts in Eq. (1). For the inclusive cross sections elastic, quasi-elastic, and inelastic processes need to be taken into account, whereas for the semi-inclusive ones only inelastic radiative processes contribute. The correction for the ratio of the latter was taken to be independent of $z$. Since the inelastic radiative effects are almost the same for the nuclei $A$ and $D$, the size of the radiative corrections applied to $R_{A}^{h}$ was small over most of the kinematic range. Only in kinematic regions of DIS where the elastic and quasi-elastic tails are non-negligible, i.e., at the highest value of $v$ and lowest value of $Q^{2}$ (low $x_{\mathrm{Bj}}$ ), is there a noticeable effect on $R_{A}^{h}$, with a maximum (increase) of $R_{A}^{h}$ of about $7 \%$ for xenon and krypton, $4.5 \%$ for neon, and $1 \%$ for helium.

Since the usual interpretation of the definition of $R_{A}^{h}$ (see Eq. (1)) is that it should only include hadrons formed in the fragmentation process, a correction has to be made for measured hadrons that are the decay products of directly produced mesons. The main effect is on the charged-pion multiplicities as a result of the decay of exclusively produced $\rho^{0}$ mesons (for pions from other mesons and for other hadrons the contribution is small). That may affect the multiplicities for 
positive (negative) pions by an amount ranging from about $1 \%$ at low $z$ up to $30 \%(45 \%)$ at high values of $z$, as estimated from a Monte Carlo simulation. The effect on the multiplicity ratio $R_{A}^{\pi}$ is much smaller, but does not cancel completely since the $\rho^{0}$ mesons also interact with the nuclear medium. Taking into account the measured nuclear transparency [39] for $\rho^{0}$ mesons, the maximum remaining effect on $R_{A}^{\pi}$, which occurs for $z$-values of $0.7-0.8$, was estimated to be about $2(4) \%, 3(5) \%, 3.5(6) \%$, and 4(7)\% in the case of helium, neon, krypton, and xenon, respectively. The first(second) number applies for positive (negative) pions. These values were included in the systematic uncertainties.

The total systematic uncertainties include the uncertainties of hadron identification (1.5\% for neutral pions, $0.5 \%$ for charged pions, $2 \%$ for kaons, $2 \%$ for protons, and $6 \%$ for antiprotons), overall efficiency $(<2 \%), \rho^{0}$-meson production for positive $(0.3 \%-4 \%)$ and negative $(0.3 \%-$ $7 \%$ ) pions, and the effects of using different parameterizations of fragmentation functions and distribution functions in the RC calculations $(<2 \%)$.

\section{Results and discussion}

In this section the experimental results are presented and the dependences of the multiplicity ratios $R_{A}^{h}$ on the various kinematic variables and the mass number $A$ of the nucleus are discussed. Unless specified otherwise the data are shown with the following constraints: $v>6.0 \mathrm{GeV}, z>$ 0.2 , and $x_{F}>0$, where $x_{F}$ is given by

$$
x_{F}=p_{\|}^{*} / p_{\|}^{* \max },
$$

with $p_{\|}^{*}$ being the component of the hadron momentum in the direction of the momentum transfer in the virtual-photon-nucleon center-of-mass system. Together with that on $z$, the constraint on $x_{F}$ will reduce possible contributions from target fragmentation.

\subsection{Multiplicity ratio for identified hadrons}

Figs. 2-5 show the dependence of $R_{A}^{h}$ on $v, z, Q^{2}$, and $p_{t}^{2}$ for the various nuclei for all identified hadrons: positively charged (pions, kaons, and protons), negatively charged (pions, kaons, and antiprotons), and neutral ones (pions). The inner error bars in these figures represent the statistical uncertainties, while the outer ones are for the total uncertainty (statistical plus systematic, added quadratically). The systematic uncertainty is mainly a scale uncertainty, affecting the values of $R_{A}^{h}$ for the various values of $v, z, Q^{2}$, or $p_{t}^{2}$ in the same way.

In presenting the results for $R_{A}^{h}$ as functions of one of the four independent variables $(v, z$, $Q^{2}, p_{t}^{2}$ ) only, $R_{A}^{h}$ was integrated (within the acceptance of the experiment) over the others. Because of acceptance effects and because in general the dependence of $R_{A}^{h}$ on $v, z, Q^{2}$, and $p_{t}^{2}$ does not factorize $\left(R_{A}^{h}\left(v, z, Q^{2}, p_{t}^{2}\right) \neq R_{1}(v) R_{2}(z) R_{3}\left(Q^{2}\right) R_{4}\left(p_{t}^{2}\right)\right)$, this integration may introduce false dependences. This is mainly relevant in case of $v$ and $z$, where the average value of $v$ ( $z$ ) changes non-negligibly depending on the value of $z(v)$. Table A.1 in Appendix A gives an indication of the size of these correlations by listing the average values of the kinematic quantities that were integrated over for the various dependences in the case of produced pions. All data are available in detail from Ref. [40].

Before discussing in the following subsections the dependence of $R_{A}^{h}$ on the kinematic variables $v, z, Q^{2}$, and $p_{t}^{2}$ in detail, first some global features of the data are discussed.

The basic feature of the data is the decrease of $R_{A}^{h}$ with increasing value of the mass number $A$ of the nucleus. Qualitatively this is understood as being due to increased partonic (quark energy 


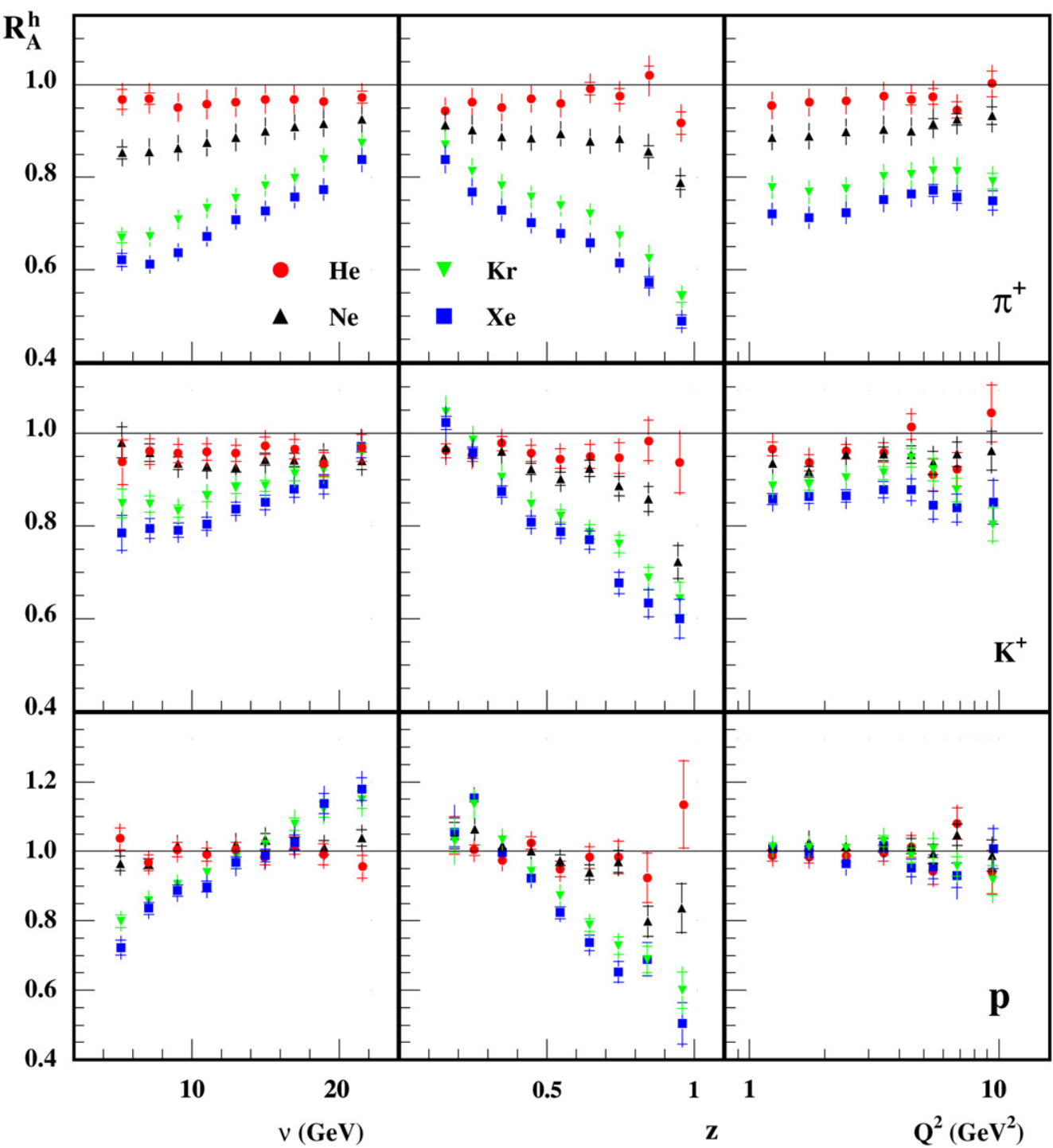

Fig. 2. Values of $R_{A}^{h}$ for positively charged hadrons as a function of $v, z$, and $Q^{2}$. The data as a function of $v$ are shown for $v>4 \mathrm{GeV}$ and those as a function of $z$ for $z>0.1$. The inner error bars represent the statistical uncertainty, while the outer ones show the total uncertainty.

loss) or hadronic (absorption) effects. Furthermore, there is a large similarity between the data for $\pi^{+}, \pi^{-}$, and $\pi^{0}$, and a clear difference between those for $K^{+}$and $K^{-}$, and those for $p$ and $\bar{p}$. Also here there are some simple arguments to explain these features at least qualitatively.

Since we use (almost) isoscalar targets and the production of $\pi^{+}$and $\pi^{-}$on protons or neutrons is only slightly different, both the production and absorption of pions is in the first instance independent of their charge. The values of $R_{A}^{h}$ for $K^{+}$and $K^{-}$show a similar behaviour as a function of the various variables, but $R_{A}^{K^{-}}$is almost everywhere smaller than $R_{A}^{K^{+}}$. A positive kaon can be produced directly from the struck quark (in the language of string breaking mod- 


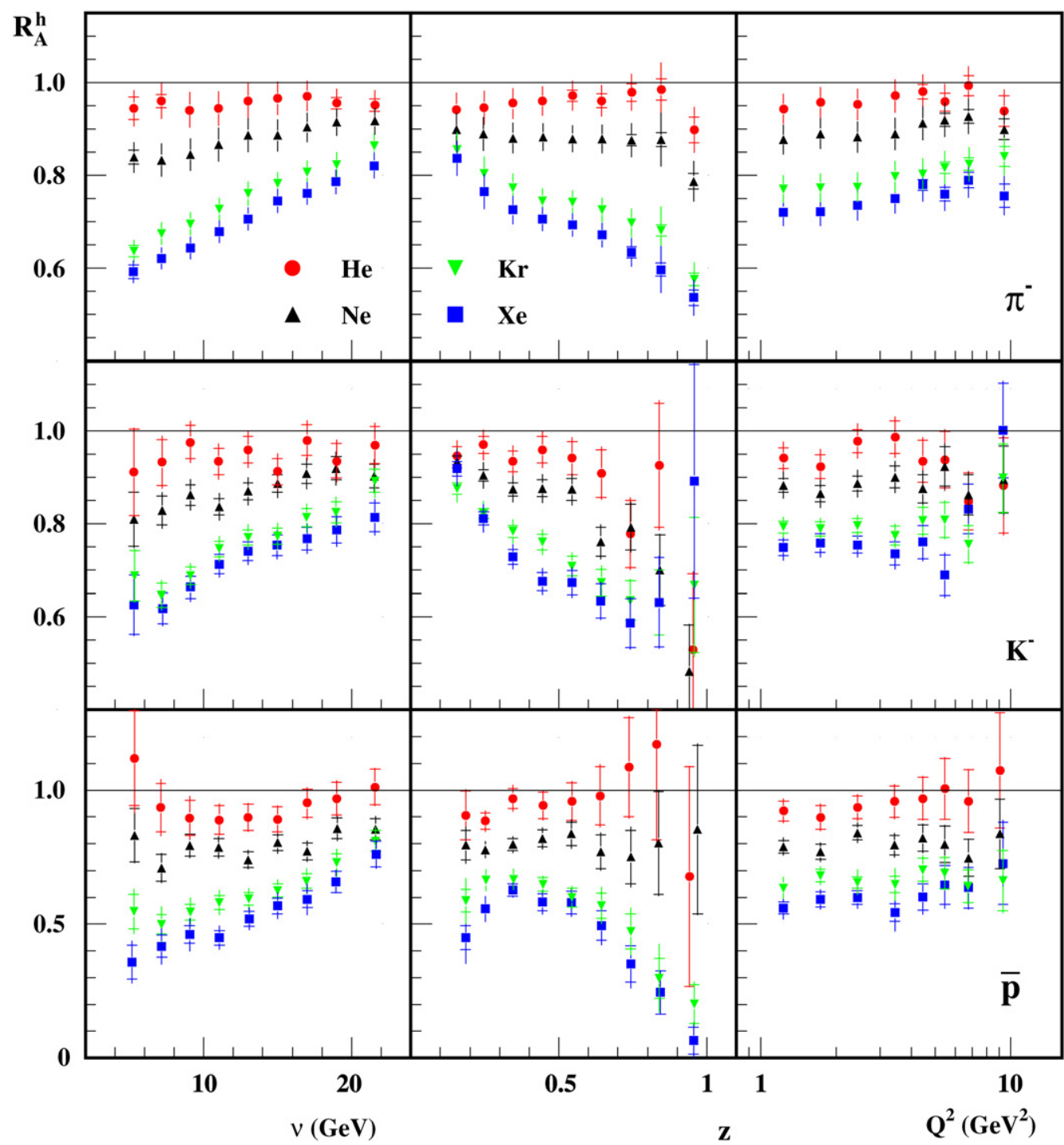

Fig. 3. Values of $R_{A}^{h}$ for negatively charged hadrons as a function of $v, z$, and $Q^{2}$. The data as a function of $v$ are shown for $v>4 \mathrm{GeV}$ and those as a function of $z$ for $z>0.1$. Error bars as in Fig. 2.

els it is a rank 1 hadron), but a negative kaon can only be produced in more complicated string breakings (rank 2 or higher), except at small values of $x_{\mathrm{Bj}}$, where sea quarks start to play a larger role. This is reflected in the production rate on deuterium being much larger for $\mathrm{K}^{+}$than for $K^{-}$, see Table 3, and leads to a steeperr dependence of the $K^{-}$fragmentation function on $z$, and hence a reduced production if the parton has lost energy before hadronization. Also, due to their quark content, nuclear absorption cross sections are larger for $K^{-}$than for $K^{+}$. Thus both parton energy loss and absorption of the produced kaon can qualitatively explain the observed difference between $R_{A}^{K^{+}}$and $R_{A}^{K^{-}}$. However, when comparing the multiplicity ratios for pions and kaons, it is seen that $R_{A}^{K^{-}} \approx R_{A}^{\pi^{-}}$, whereas $R_{A}^{K^{+}}>R_{A}^{\pi^{+}}$. Given that both pions and $K^{+}$ 


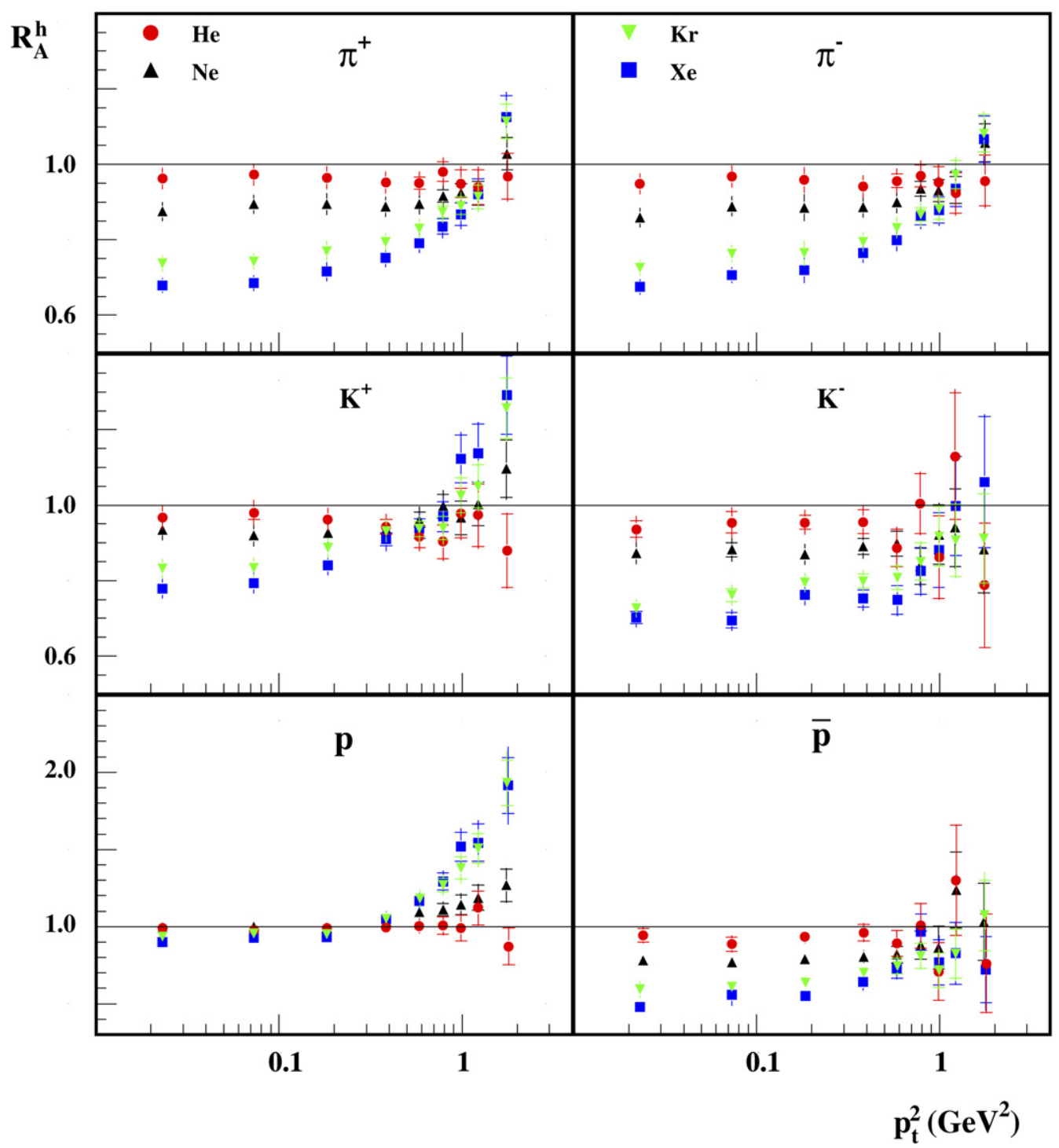

Fig. 4. Values of $R_{A}^{h}$ for positively (left panel) and negatively (right panel) charged hadrons as a function of $p_{t}^{2}$. Error bars as in Fig. 2.

particles are rank-1, and $K^{-}$rank 2 or higher, and that nuclear absorption cross sections for both $K^{+}$and $K^{-}$are smaller than for pions, these features are not readily explained by the behaviour of fragmentation functions or absorption cross sections.

The results for protons cannot really be related to those for any of the other particles. Because protons are already present in a nucleus, an appreciable fraction of them may not come from hadronization. This is reflected in the very large difference in production of $p$ and $\bar{p}$ on deuterium, see Table 3. Furthermore, as discussed in Section 2, in final-state interactions they 


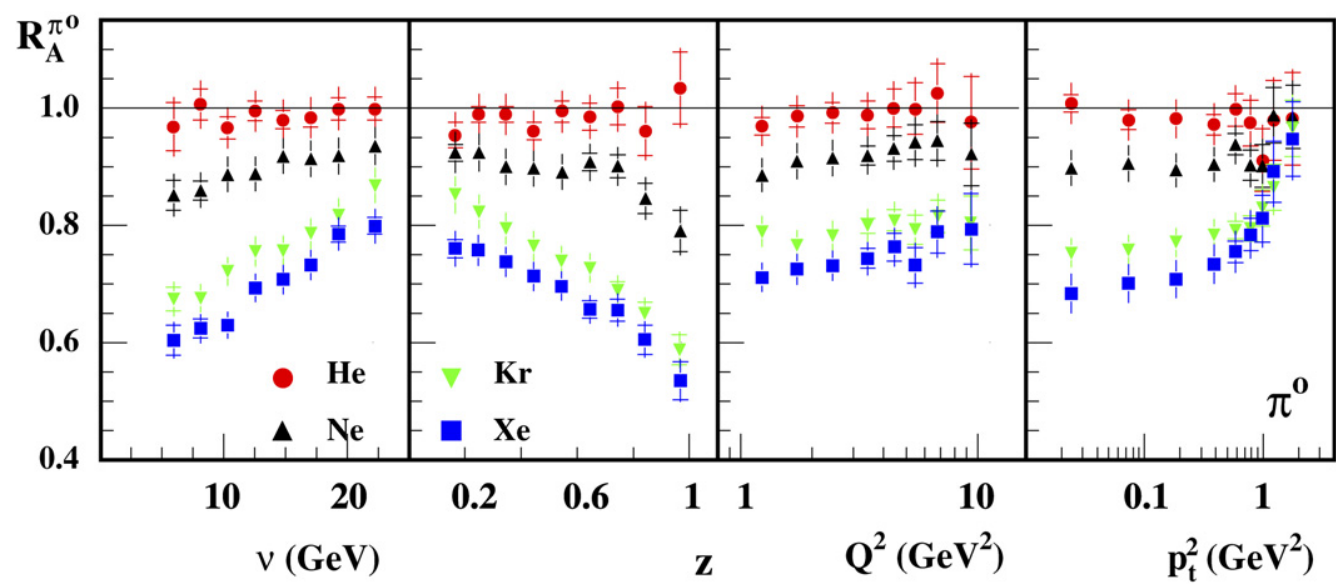

Fig. 5. Values of $R_{A}^{h}$ for neutral pions as a function of $v, z, Q^{2}$, and $p_{t}^{2}$. The data as a function of $z$ are shown for $z>0.1$. Error bars as in Fig. 2.

generally are not absorbed, but give rise to more nucleons (both protons and neutrons), thus possibly even increasing $R_{A}^{h}$ at lower $z$.

Antiprotons feature a rather strong attenuation, which might be attributed to the relatively large $\bar{p} N$ cross section.

\subsection{1. v-dependence}

The first systematic experimental study of the $v$-dependence of attenuation was reported in Ref. [2], where the range $20<v<200 \mathrm{GeV}$ was investigated. It was shown that the nuclear attenuation decreased with increasing value of $v$, and essentially vanished at $v>50 \mathrm{GeV}$. The HERMES data are more informative, since they are in the region of $v$ where the attenuation becomes appreciable, include particle identification, and the statistical precision is much better.

The leftmost columns in Figs. 2, 3, and 5 show that in almost all cases the attenuation decreases (the value of $R_{A}^{h}$ increases) with increasing values of $v$. (For He this behaviour presumably is present as well, but small compared to the uncertainties in the data points.) In the absorption-type models this is explained as being due to an increase of the formation length in the rest frame of the nucleus at higher $v$ due to Lorentz dilatation, resulting in a larger fraction of the hadronization taking place outside the nucleus. In partonic models the quark energy loss leads effectively to a shift $\Delta z$ in the argument of the fragmentation function, and thus an attenuation that is proportional to $\epsilon / \nu$, with $\epsilon$ the quark energy loss.

For protons $R_{A}^{h}$ increases at higher values of $v$ to well above unity for $\mathrm{Kr}$ and $\mathrm{Xe}$. Here the following should be realized. The value of $\langle z\rangle$ is correlated with $v$, e.g., the value of $\langle z\rangle$ for the lowest $v$-bin is about 0.57 , whereas for the highest $v$-bin it is 0.35 . Since the value of $R_{A}^{h}$ strongly increases with decreasing value of $z$ (see the next subsection), a large fraction of the strong increase at high $v$ is in fact due to the dependence of $R_{A}^{h}$ on $z$. Such an effect may play a role for other particles, e.g., for $K^{+}$, too.

\subsection{2. z-dependence}

As can be seen from the second column in Figs. 2, 3, and 5 for all hadron types $R_{A}^{h}$ is largely constant with $z$ for He, while it decreases with increasing $z$ for $\mathrm{Ne}$ and especially for Xe and $\mathrm{Kr}$. In parton energy-loss models this results from the strong decrease of the fragmentation function 
at large $z$ in combination with the $\Delta z$ resulting from the energy loss. In absorption-type models the overall decrease of $R_{A}^{h}$ with increasing $z$ is assumed to be due to a decrease in the formation length in combination with (pre)hadronic absorption. The increase of $R_{A}^{h}$ at large $z$ calculated in Ref. [22] is not observed in the data.

For the heavier targets $R_{A}^{h}$ rises strongly at low $z$. Presumably this is due to large FSI effects in these nuclei, through which particles of higher energy lose energy or get absorbed, generating (other) lower-energy particles.

As in the case of large values of $v$, the value of $R_{A}^{h}$ for protons rises above unity at small $z$. This presumably is a result of large rescattering of protons and other produced particles with protons in the target. Part of the increase is due to the fact that the average value of $v$ decreases considerably with $z$, from about $18 \mathrm{GeV}$ for the lowest bin to about $10 \mathrm{GeV}$ for the highest bin. This explains also why even at the smallest $z$ the value of $R_{A}^{h}$ is still lower than that for the highest value of $v$.

Apart from featuring rather small values of $R_{A}^{h}$, down to almost zero, the $z$-dependence of $R_{A}^{h}$ for antiprotons is special in that it stays constant or even decreases slightly at small values of $z$, where $R_{A}^{h}$ for other particles increases strongly for the heavier targets. This probably can be attributed to the fact that in final-state interactions the chance that an antiproton survives, or is produced, is relatively small. This would support the idea that the rise of $R_{A}^{h}$ for other particles at $z<0.3$ is due to FSI effects and suggests that the difference in behaviour of antiprotons and the other particles at small values of $z$ may be a sensitive check on coupled-channel calculations of FSI effects.

\subsection{3. $Q^{2}$-dependence}

The rightmost column of Figs. 2, 3, and 5 shows for pions a small $Q^{2}$-dependence, which is slightly stronger for the heavier nuclei. For kaons and (anti-)protons no $Q^{2}$-dependence is discernable. Hence, the attenuation is not very sensitive to $Q^{2}$, which means that integrating over $Q^{2}$ when studying other dependences does not introduce false dependences.

In the twist-4 energy-loss model of Refs. [20,21] a $Q^{2}$-dependence of $R_{A}^{h}$ of the form $R \sim a \ln Q^{2}$ is found, which is consistent with the data. In Ref. [26] the calculated $Q^{2}$ dependence is the result of two counteracting processes, which results in a rather small $Q^{2}$ dependence that is larger for $\mathrm{Kr}$ than for $\mathrm{Ne}$, in global agreement with the data. When describing the attenuation purely as the result of a modification of the effective fragmentation function [22], a slight increase of $R_{A}^{h}$ with $Q^{2}$ is predicted. The deconfinement model [25] predicts for all nuclei a slight decrease of $R_{A}^{h}$ with $Q^{2}$, which is not supported by the data.

\subsection{4. $p_{t}^{2}$-dependence}

Fig. 4 and the rightmost column of Fig. 5 show for the heavier nuclei a rise of $R_{A}^{h}$ at high $p_{t}^{2}$. Such an effect was first observed by EMC [2] for all charged hadrons taken together, but has been measured now for separate identified hadrons. The phenomenon is also known from heavyion collisions, where it is referred to as the Cronin effect [41]. Compared to experiments with heavy ions, the use of a lepton probe has the advantage that initial-state interactions do not play a role, except for shadowing effects, which are small in the $x_{\mathrm{Bj}}$ range of the present experiment. The observed rise at high $p_{t}^{2}$ is attributed to a broadening of the $p_{t}^{2}$ distribution. In principle this can result from partonic rescattering as well as from hadronic final-state interactions. In the next subsection this will be looked at in more detail in the case of pions. 


\subsection{Two-dimensional multiplicity ratio for pions}

In order to investigate the behaviour of $R_{A}^{h}$ in more detail, the data for $\pi^{+}$and $\pi^{-}$production, which have the best statistical precision and are consistent within uncertainties, were combined for a two-dimensional binning, see Figs. 6-8. The bins used are 0.2-0.4-0.7-1.2 for $z$, 6.0-12.0-17.0-23.5 GeV for $v$, and smaller or larger than $0.7 \mathrm{GeV}^{2}$ for $p_{t}^{2}$. This has the added advantage that the correlation between, e.g., the average values of $v$ and $z$ mentioned in relation to the one-dimensional distributions, is strongly reduced.

Fig. 6 shows the dependence of $R_{A}^{\pi}$ on $v, Q^{2}$, and $p_{t}^{2}$ for three bins in $z$. The left hand column indicates that the dependence on $v$ hardly depends on $z$. The $Q^{2}$-dependence is similar for the various $z$-bins (Monte Carlo simulations show that the rise of $R_{A}^{\pi}$ at the lowest $Q^{2}$ for the highest $z$ range is due to a relatively large contribution of pions coming from $\rho^{0}$ decay). Therefore, the dependence on $z$ is not affected when integrating over $Q^{2}$. The data in the rightmost column indicate that the increase of $R_{A}^{h}$ for $\mathrm{Kr}$ and $\mathrm{Xe}$ at large $p_{t}^{2}$ is smaller for larger $z$. Such a $z$-dependence of the $p_{t}^{2}$-dependence was predicted in Ref. [26]. The points for the highest $z$ range show a bump at small values of $p_{t}^{2}$. This is due to a relatively large contribution of pions coming from $\rho^{0}$ decay in this part of the phase space.

Fig. 7 shows the dependence of $R_{A}^{\pi}$ on $z, Q^{2}$, and $p_{t}^{2}$ for three bins in $v$. The second and third column indicate that the dependence of $R_{A}^{h}$ on $Q^{2}$ and $p_{t}^{2}$ depends only weakly on $v$, which allows one to integrate over these variables without introducing spurious correlations. The leftmost column shows for $\mathrm{He}$ and $\mathrm{Ne}$ (and perhaps for $\mathrm{Kr}$ ) some interesting features in that there seems to be a change of the $z$-dependence with the value of $\nu, R_{A}^{\pi}$ first being about constant or even rising slightly with $z$, and then dropping, the turnover point occurring at lower $z$ for the lower $v$-bin. This behaviour is studied in more detail in the next subsection.

Fig. 8 shows the dependence of $R_{A}^{\pi}$ on $v, Q^{2}$, and $z$ for two bins in $p_{t}^{2}$. The leftmost column shows that at large $p_{t}^{2}$ the values of $R_{A}^{h}$ are larger and that the dependence on $v$ largely disappears. This is clearly correlated with the fact that $R_{A}^{h}$ increases at large $p_{t}^{2}$ (Cronin effect), as discussed in the previous subsection, and indicates that this effect is largely independent of $v$. The rightmost column of this figure shows that the Cronin effect disappears at high $z$. This is at least consistent with the idea that the rise of $R_{A}^{h}$ at large $p_{t}^{2}$ (broadening of the $p_{t}^{2}$-distribution) is of partonic origin. In the limit $z \rightarrow 1$ there is no room for partonic rescattering, because the parton is not allowed to have any energy loss (see, e.g., Ref. [26]). In principle, rescattering of the produced (pre)hadron could lead to the observed behaviour, too, but since the rescattering cross sections are relatively small, the data discussed suggest a partonic mechanism. At the same time, this tells that the attenuation in the limit $z \rightarrow 1$ is due purely to a hadronic absorption mechanism.

\subsection{Dependence of $R_{A}^{\pi}$ on formation length}

Given the ideas of how hadronization proceeds in time, as e.g., in the Lund model, the formation length, which depends on both $v$ and $z$, may be a more efficient variable for describing the kinematic dependence of $R_{A}^{h}$ than $v$ and $z$. This idea was recently pursued in Ref. [42]. In order to investigate this, values of $R_{A}^{\pi}$ versus $L_{c}$ for various values of $v$ and $z$ are shown in Fig. 9. Here Eq. (2) is used with

$$
f(z)=z^{0.35}(1-z)
$$



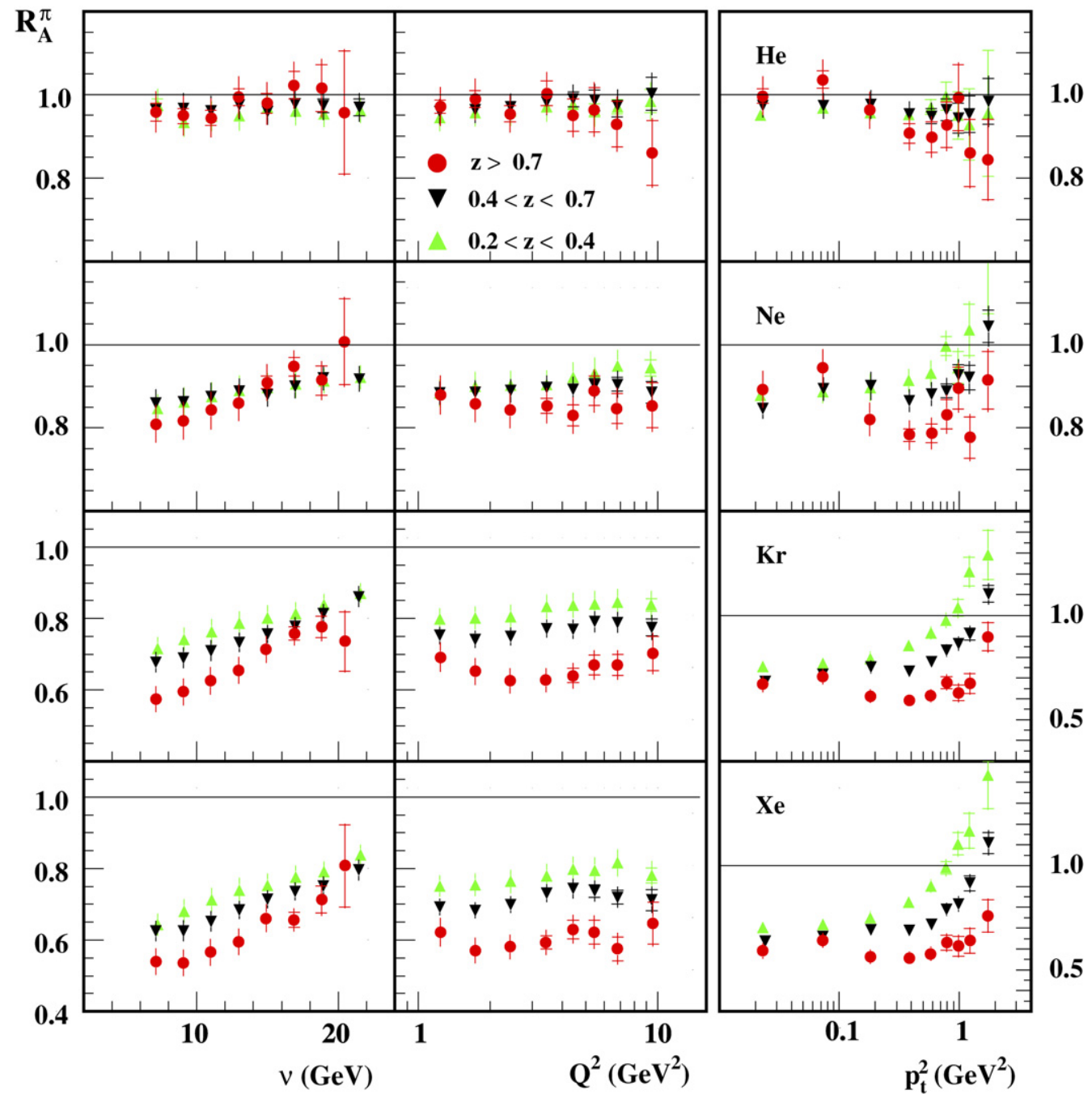

Fig. 6. Values of $R_{A}^{h}$ for charged pions for three $z$ ranges. Error bars as in Fig. 2.

and $\kappa=1 \mathrm{GeV} / \mathrm{fm}$. This form for $f(z)$ is a convenient parametrization obeying the constraints at $z \rightarrow 0$ and $z \rightarrow 1$, and gives values for $L_{c}$ as a function of $z$ closely resembling the ones obtained with the Lund model.

A clear correlation can be observed between the values of $R_{A}^{h}$ and $L_{c}$, with only a relatively small residual spread at any fixed value of $L_{c}$. Evidently most of the dependence of $R_{A}^{h}$ on $v$ or $z$ in Figs. 6-7 can be described as a dependence on $L_{c}$, which thus acts as a scaling variable. Upon close inspection it is seen that for almost all $v$ values the data points for the lowest two $z$ values (the two rightmost in the sequence of same symbols) bend slightly upwards. This behaviour is most likely due to large rescattering effects in the lowest $z$-bin (note the relatively strong rise of $R_{A}^{\pi}$ at low $z$ in Figs. 2 and 3). 

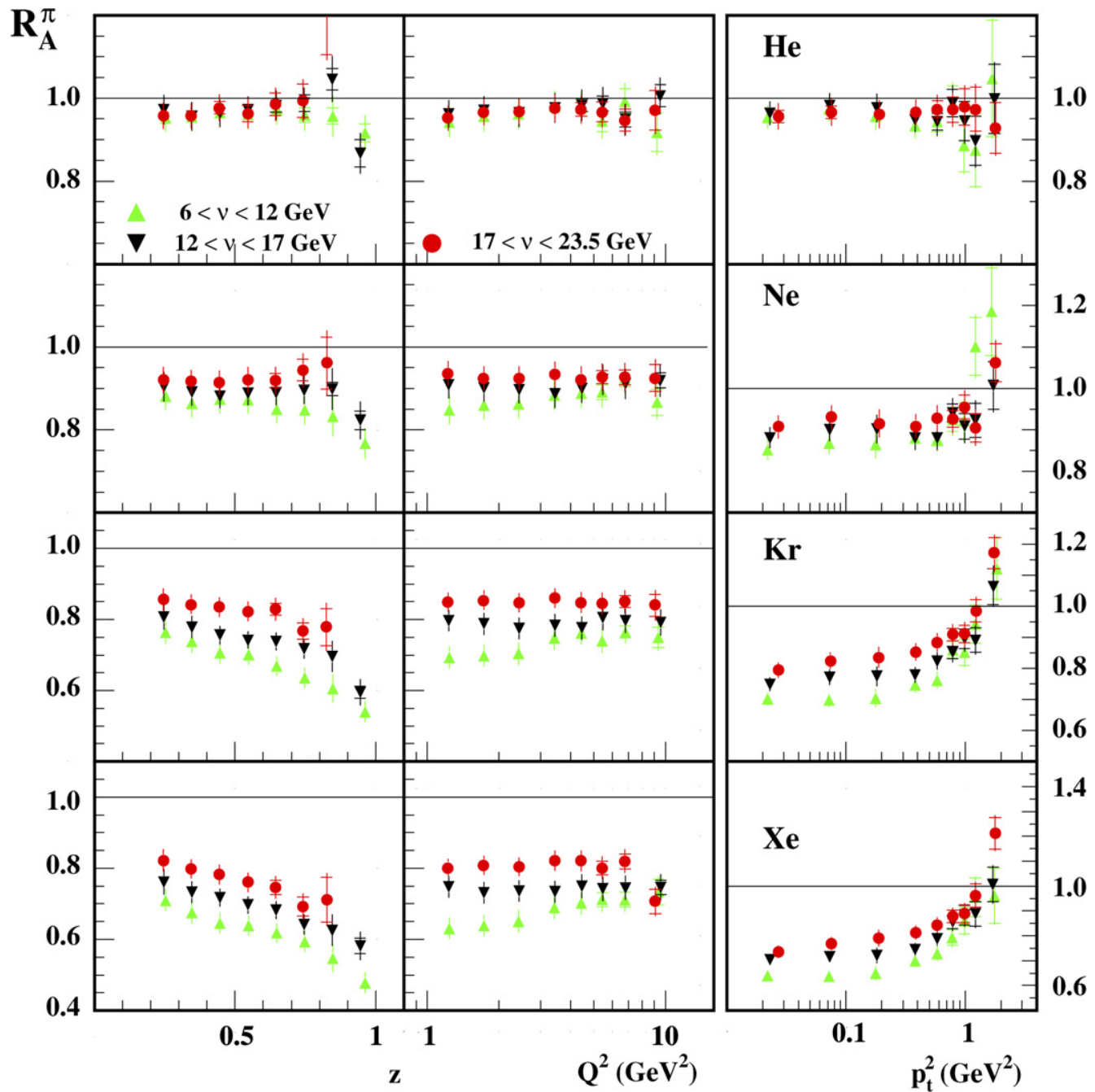

Fig. 7. Values of $R_{A}^{h}$ for charged pions for three $v$ ranges. Error bars as in Fig. 2.

For He the value of $R_{A}^{\pi}$ rises for the first few points at small $L_{c}$ and then becomes constant. For $\mathrm{Ne}$ the initial rise extends over more points, and changes then to a much more gradual one. For $\mathrm{Kr}$ and $\mathrm{Xe}$ the change in slope is much more gradual, but still noticeable. This is illustrated by the two straight lines in the plot for Xe, which represent fits to the data for the ranges $L_{c}<4 \mathrm{fm}$ and $L_{c}>5 \mathrm{fm}$. This suggests the following interpretation: at the larger values of $L_{c}$, which are (much) larger than the size of these nuclei, ${ }^{2}$ even if the absolute scale of $L_{c}$ may have some uncertainty because the value of $\kappa$ is not precisely known, one probably sees a partonic mech-

\footnotetext{
2 It should be realized that the average distance that a created parton travels through a nucleus (assuming it is not absorbed) is only $\frac{3}{4} R$, with $R$ the radius of that nucleus, because the virtual photon can interact anywhere in a nucleus. Thus, even for $\mathrm{Kr}$ with a radius of about $5 \mathrm{fm}$, hadronic mechanisms become small when $L_{c}>4 \mathrm{fm}$.
} 


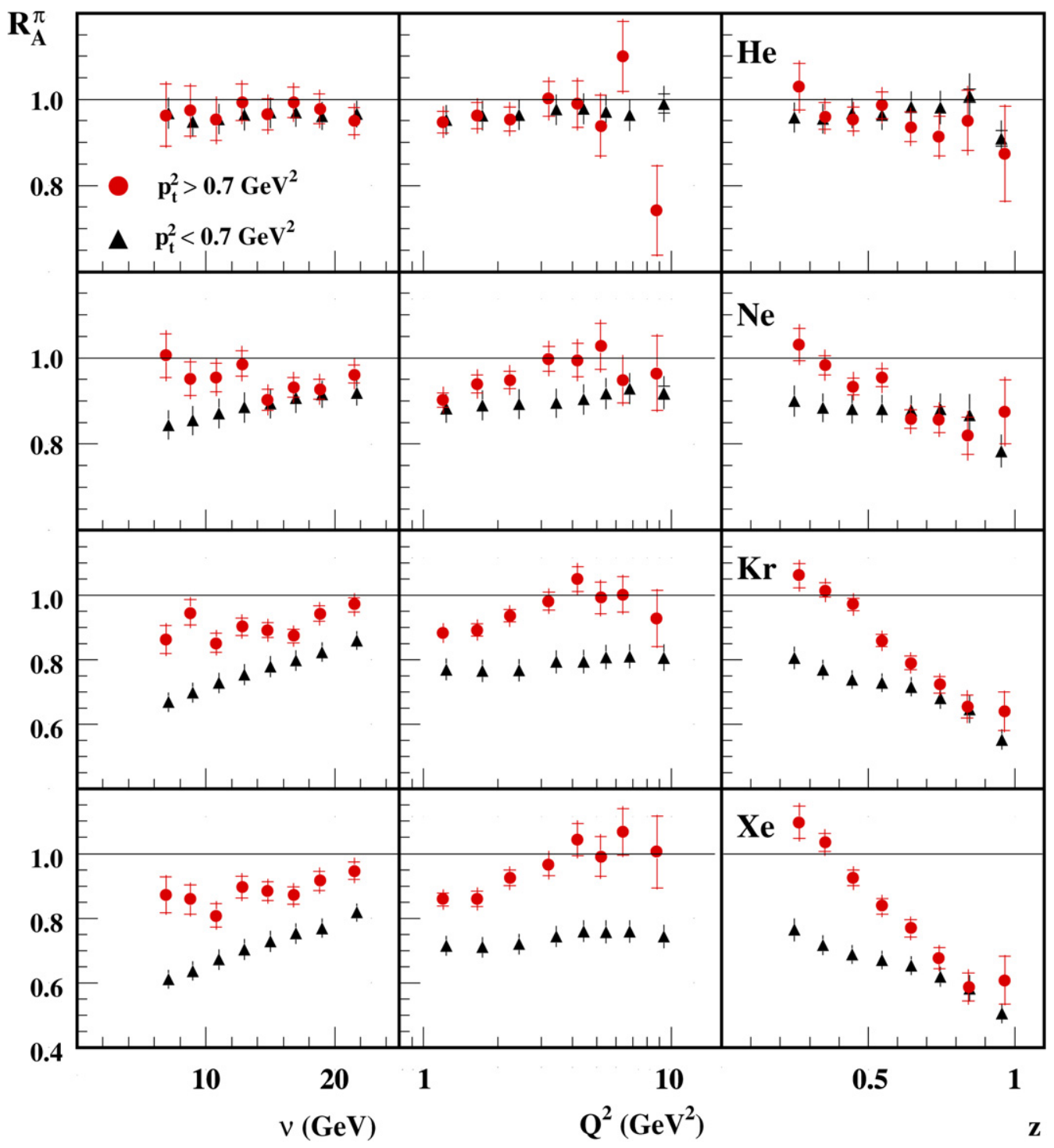

Fig. 8. Values of $R_{A}^{h}$ for charged pions for two $p_{t}^{2}$ ranges. Error bars as in Fig. 2.

anism. The data for Ne suggest that this mechanism still has a dependence on $L_{c}$, presumably through the underlying values of $v$ and $z$. The drop of $R_{A}^{\pi}$ for low values of $L_{c}$ then results most probably from hadronic mechanisms. The data on $\mathrm{Kr}$ and $\mathrm{Xe}$ are consistent with this interpretation. At low values of $L_{c}$ there is a strong attenuation (stronger in the larger nucleus Xe) due to hadronic absorption (on top of the partonic contribution). Since the partonic effect must disappear when $z \rightarrow 1$, the value of $R_{A}^{\pi}$ for $L_{c} \rightarrow 0$ at finite $v$ is purely due to the hadronic mechanism. The disappearance of the partonic contribution in this limit may be an explanation why the data for the lower values of $L_{c}$ at given value of $v$ tend to lie above the data at the same $L_{c}$ with lower $v$-value. At higher values of $L_{c}$ the influence of absorption becomes smaller, since 


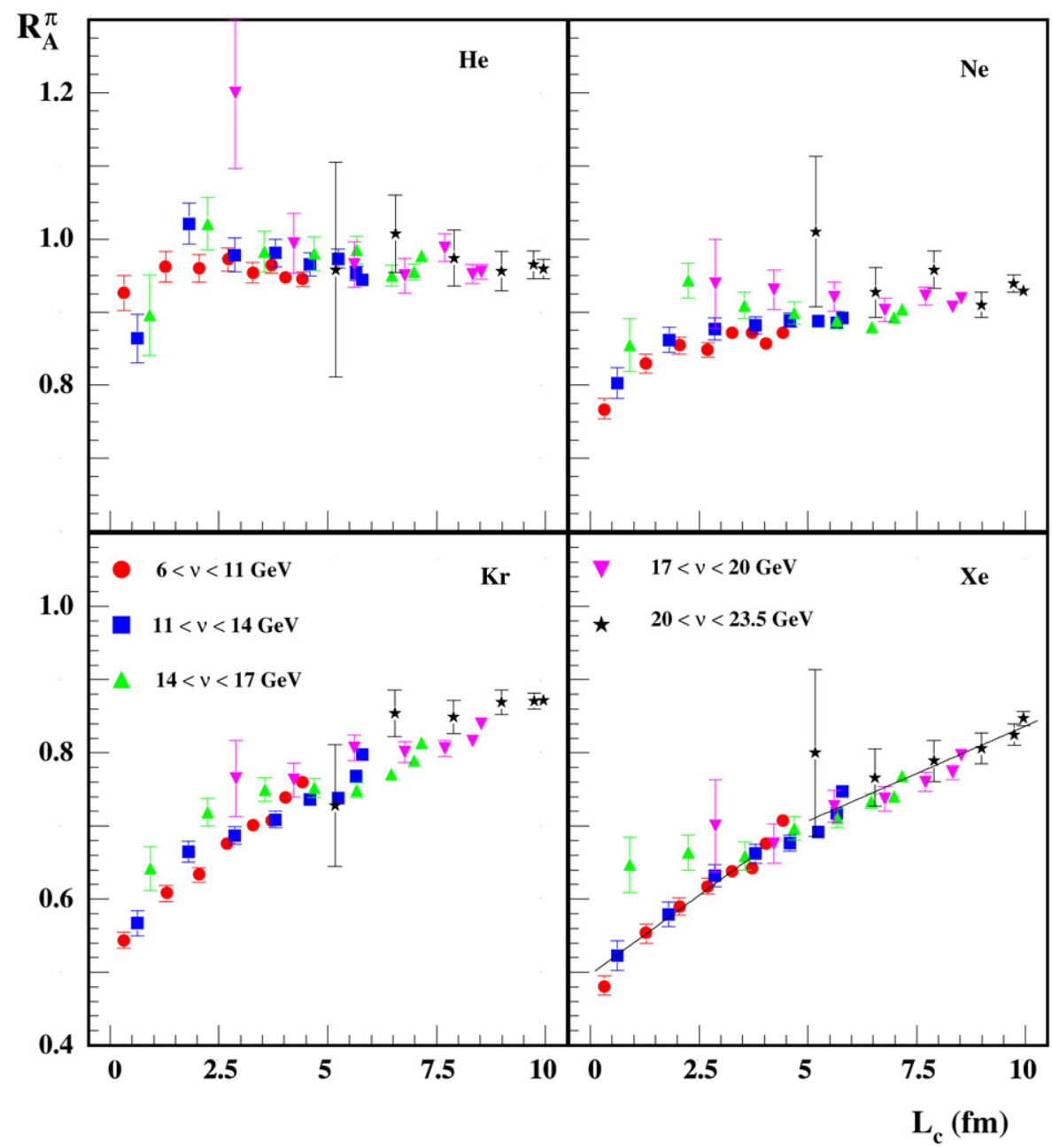

Fig. 9. Values of $R_{A}^{\pi}(v, z)$ for $\mathrm{He}, \mathrm{Kr}, \mathrm{Ne}$, and $\mathrm{Xe}$ as a function of the variable $L_{c}$, see Eqs. (2) and (4). The various $v$-bins are indicated by different symbols. Within the same $v$ bin the $z$ bins used are $0.2-0.3-0.4-0.5-0.6-0.7-0.8-0.9-1.0$, and the value of $z$ decreases from left to right. In order not to complicate the figure, only statistical error bars are shown. The systematic uncertainty is mainly a scale uncertainty of about $3 \%$.

the prehadron is increasingly produced outside the nucleus. Thus above the values of $L_{c}$ where the slope changes (below $2 \mathrm{fm}$ for $\mathrm{He}$, and around $L_{c}=2.5,4$, and $5 \mathrm{fm}$ for $\mathrm{Ne}, \mathrm{Kr}$, and $\mathrm{Xe}$, respectively), one presumably observes mainly a partonic mechanism. These tentative conclusions can only be substantiated by model calculations that include both partonic and hadronic mechanisms, and that give a good description of the measured $v$ and $z$ dependences of $R_{A}^{h}$.

\subsection{A-dependence}

The $A$-dependence of $R_{A}^{h}$ was fitted in a phenomenological way by using as the fit function

$$
R_{A}^{h}(A)=\exp \left[-\beta(A / 100)^{\alpha}\right] .
$$


The scale factor of 100 is introduced to reduce the correlation between $\alpha$ and $\beta$. At the same time the value of $\beta$ is representative now of the attenuation of a nucleus with $A=100$. For moderate values of $\beta A^{\alpha}$ this formula is equivalent to $1-R_{A}^{h}=\beta A^{\alpha}$, a parametrization that has been used before [1], but the present form has the advantage that $R_{A}^{h}$ does not become unphysical for very large values of $A$.

However, since $R_{A}^{h}$ is the ratio of the multiplicity in nucleus $A$ vs. the multiplicity in the deuteron, the fit function of Eq. (5) is internally inconsistent, unless one assumes no attenuation in the deuteron. Assuming that the attenuation depends on the (average) density times the radius of the nucleus, in Ref. [43] it is found that the attenuation in a deuteron can be described with an effective $A$-value of about 0.6. The influence of this on the value of $\alpha$ obtained using Eq. (5) is fairly small (about -0.07). Therefore, all results to be discussed in the following are based on Eq. (5), but in drawing conclusions it should be realized that the real value of $\alpha$ is probably slightly lower. More information on the performed fits with different type of fitting functions can be found in [44].

A second remark concerns what $A$-dependence is expected from different types of models for the attenuation. As already mentioned in Section 2, the attenuation in the parton energy-loss model of Refs. [20,21] is given as $1-R_{A} \sim L^{2} \sim A^{2 / 3}$, whereas in (Glauber type) absorption models it is often presumed that $1-R_{A} \sim L \sim A^{1 / 3}$. However, these estimates are too simple. Taking realistic matter distributions of nuclei yields effective values of $L^{2}$ that are proportional to $A^{0.74}$ [43]. Furthermore it was demonstrated already in Ref. [25] that the inclusion of a distribution for the formation length in absorption calculations increases the exponent above the value of $1 / 3$, yielding values for $1-R_{A}$ that approximately follow an $A^{2 / 3}$ pattern for large values of $L_{c}$, if the nucleus is described as a sphere with constant density. Using realistic matter distributions the authors of Ref. [43] find the exponent of $A$ to be $0.40,0.54$, and 0.60 when $L_{c}=0$, 2, and $4 \mathrm{fm}$, respectively.

In the fits only the statistical uncertainties in the values of $R_{A}^{h}$ have been used, as the systematic uncertainty in the values of $R_{A}^{h}$ is largely a scale uncertainty. The influence of the latter on the value of $\alpha$ was found to be about 0.05 .

\subsubsection{Pions}

First the $A$-dependence of pion production was studied as a function of $v$ and $z$, using the data binned in both $z$ and $\nu$. The results for $\alpha$ as a function of $z$ for the various $\nu$-bins are shown in Fig. 10. The values of $\beta$ (not shown) reflect the global behaviour of $R_{A}^{\pi}$ (more attenuation at higher $z$ and lower $v$ ) that is visible from earlier figures. There is an increase of $\alpha$ from about 0.5 to 0.6 with $z$ and possibly at higher $z$ a slight increase with $v$. However, in the latter region the results may be influenced by contributions from the decay of the $\rho^{0}$. The results for the highest $z$-bin do not follow the pattern of the lower $z$-bins. This is due to the behaviour of $R_{A}^{h}$ for especially $\mathrm{He}$ and $\mathrm{Ne}$, seen in Fig. 7. As mentioned before, this could result from the formation length becoming so small that even in $\mathrm{He}$ and $\mathrm{Ne}$ hadronic mechanisms become important.

Given these results for the separate $\nu$-and $z$-dependences it has also been investigated how $\alpha$ and $\beta$ depend on the value of $L_{c}$. For that purpose the data were binned in five $L_{c}$-bins, using the values of $v$ and $z$ of each event in Eqs. (2) and (4). In order to avoid possible contributions of the $\rho^{0}$ at high $z$ and large rescattering effects at low $z$, only data with $0.3<z<0.7$ were used. The resultant values of $\alpha$ and $\beta$ are shown in Fig. 11. The behaviour of $\beta$ reflects the smaller attenuation at higher $L_{c}$ already visible in Fig. 9. Consistent with what was found above, the value of $\alpha$ decreases from about 0.6 at small $L_{c}$ to less than 0.5 at large $L_{c}$. 


\section{RAPID COMMUNICATION}

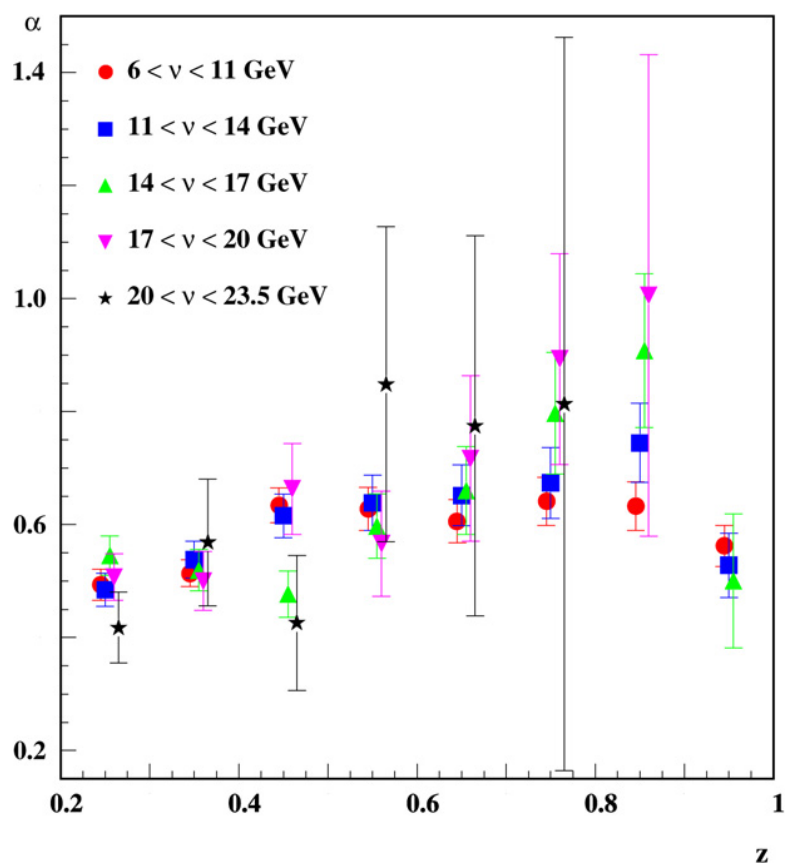

Fig. 10. Dependence of the parameter $\alpha$ (see Eq. (5)) on the value of $v$ and $z$ for the combined sample of charged pions. Points for different values of $v$ are slightly offset in $z$ for better visibility.

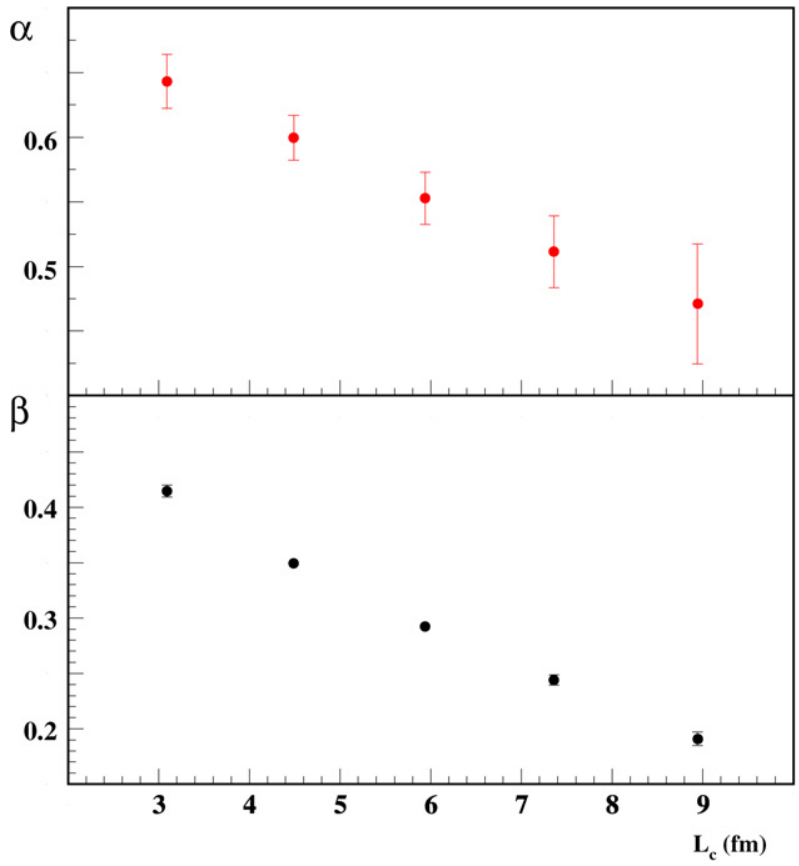

Fig. 11. The dependence of the parameters $\alpha$ and $\beta$ on the value of $L_{c}$ for $0.3<z<0.7$. 


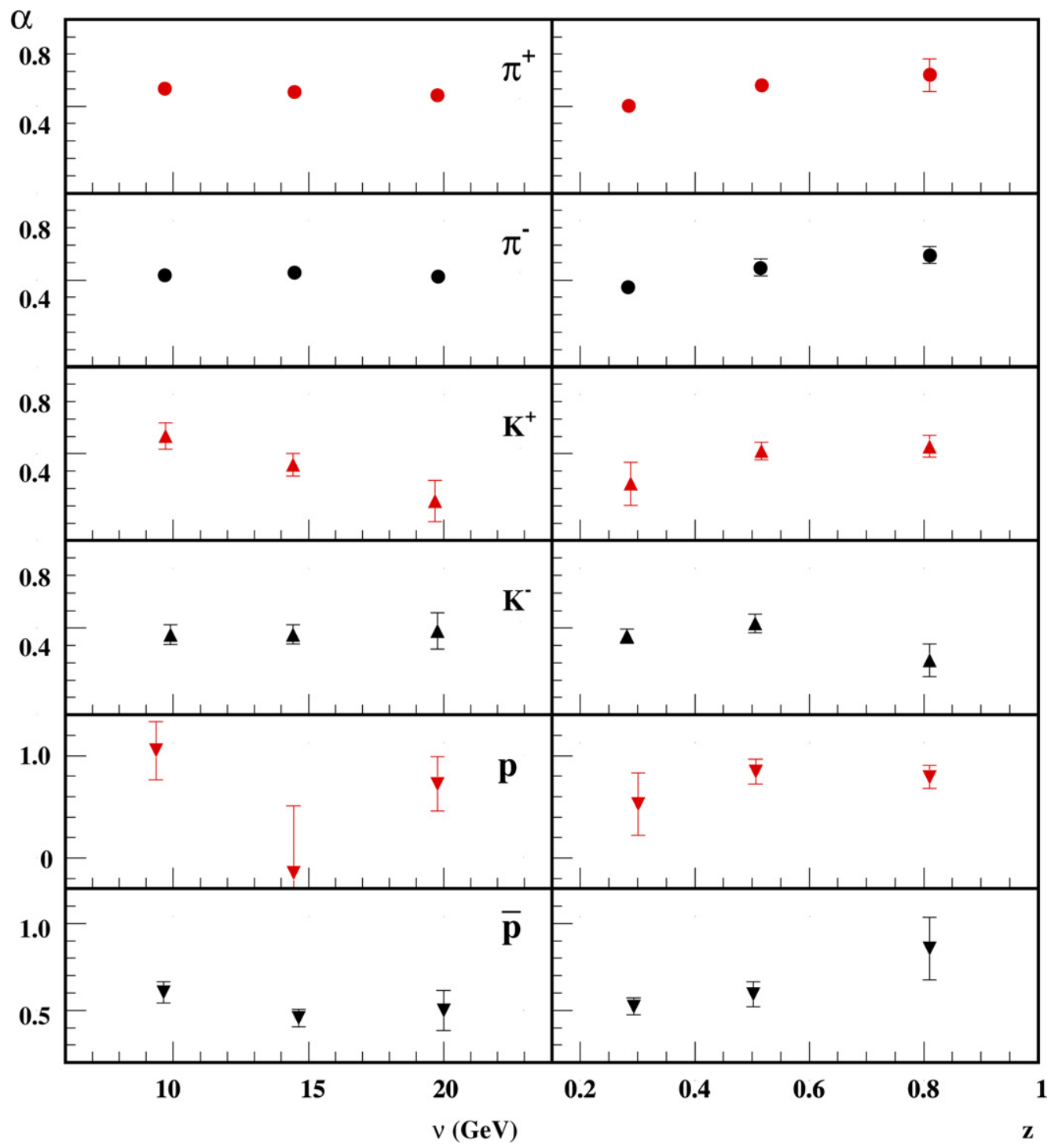

Fig. 12. Values of the parameter $\alpha$ for pions, kaons, and (anti-)protons in three $v$ ranges (left six panels), and in three $z$ ranges (right six panels).

If, as was argued in the previous subsection, partonic effects are most prominent at large values of $L_{c}$, this would indicate that the $A$-dependence of that mechanism has a value of $\alpha$ well below the value of $2 / 3$ given in Refs. [20,21] and [22].

The decrease of $\alpha$ with $L_{c}$ is not easily explained. As demonstrated in Refs. [25,43] a pure absorption mechanism would yield a value of $\alpha$ that increases with $L_{c}$. Possibly such an increase is more than compensated for by an increasing influence of the partonic mechanism. However, then that mechanism should have an $A$-dependence with a rather small value of $\alpha$. But, when two (or possibly even more) mechanisms contribute, the $A$-dependence probably becomes more 
complicated than can be described by a single exponential as in Eq. (5). For that reason comparisons between data and model calculations should be done on the level of the multiplicity ratio $R_{A}^{h}$.

\subsubsection{Other particles}

For the other particles the statistical precision is too low for a meaningful two-dimensional binning and study of the $A$-dependence, so there the $A$-dependence is presented for $\nu$-bins of 6.0-12.0-17.0-23.5 GeV (integrated over all $z$ ), and for $z$-bins of $0.2-0.4-0.7-1.2$ (integrated over all $v$ ). For comparison the values for $\pi^{+}$and $\pi^{-}$in the same bins are given as well. The results are shown in Fig. 12. The only significant feature is the different behaviour seen for $K^{+}$particles at high $v$. As mentioned before, this may be due to large rescattering effects. The behaviour for $K^{-}$is different from the one for $K^{+}$, and more like that of $\pi^{-}$, but with smaller values for $\alpha$.

\section{Summary and conclusions}

Data for the multiplicity ratio $R_{A}^{h}$ of hadron production in semi-inclusive deep-inelastic scattering of $27.6 \mathrm{GeV}$ electrons and positrons from helium, neon, krypton, and xenon nuclear targets relative to deuterium were obtained for identified $\pi^{+}, \pi^{-}, \pi^{0}, K^{+}, K^{-}, p$, and $\bar{p}$ particles as a function of the virtual-photon energy $\nu$, the fraction $z$ of the energy transferred to the hadron, the photon virtuality $Q^{2}$, and the hadron transverse momentum squared $p_{t}^{2}$. For all particles the dependence of $R_{A}^{h}$ on these variables is presented and discussed.

The most prominent features of the data are an increased attenuation (decrease of $R_{A}^{h}$ below unity) with increasing value of the mass number $A$ of the nucleus and the attenuation becoming smaller (larger) with increasing values of $v(z), R_{A}^{h}$ dropping below 0.5 for xenon in some kinematic regions. At low values of $z$, especially for heavier targets and for protons and $K^{+}$particles, a strong rise of $R_{A}^{h}$, even to above unity, is observed. Presumably this is due to hadronic rescattering, where a higher energy particle through nuclear reactions produces one or more lower-energy particles.

The value of $R_{A}^{h}$ increases slightly with $Q^{2}$, at least for pions, and is almost independent of $p_{t}^{2}$, except at large values of $p_{t}^{2}$, where $R_{A}^{h}$ increases strongly. The latter is thought to result from $p_{t}^{2}$ broadening due to partonic rescattering (Cronin effect). This effect was seen to disappear for $z \rightarrow 1$, in accordance with the picture that in that limit no rescattering is possible, since rescattering of the struck parton implies an energy loss.

By combining the data for $\pi^{+}$and $\pi^{-}$, the dependence of $R_{A}^{\pi}$ on two of the variables $v, z$, $Q^{2}$, and $p_{t}^{2}$ together was investigated. The dependence on $Q^{2}$ depends weakly but noticeably on the value of $v$, but practically not on that of $z$. The dependence on $p_{t}^{2}$ hardly depends on $v$ and $z$, except for the disappearance of the rise at large $p_{t}^{2}$ at $z \rightarrow 1$ mentioned above. However, the dependences on $v$ and $z$ are related. It was found that most of the dependence on $v$ and $z$ can be incorporated in a dependence on the combination $L_{c}=z^{0.35}(1-z) v / \kappa$, where $\kappa$ is the string tension in string models, which thus acts as a scaling variable. Since this function is close to the one given in the Lund model for the average formation length of a particle, by inspecting the value of $R_{A}^{\pi}$ vs. $L_{c}$ for the four nuclei, regions can tentatively be identified, where hadronic (absorption) plus partonic mechanisms are important, and a region at higher $L_{c}$ where only or mainly partonic mechanisms play a role.

A fit of the $A$-dependence of the values of $R_{A}^{\pi}$ for pions measured for the various nuclei of the form $R_{A}^{\pi}=\exp \left[-\beta(A / 100)^{\alpha}\right]$ yields values of $\alpha$ from about 0.6 to 0.5 , depending on the 
value of $L_{c}$. Similar values are found for the other particles. These values of $\alpha$ are well below the values resulting from models in which the attenuation depends on the square of the distance a parton travels through the nucleus.

In total a very extensive data set to guide modeling hadronization in nuclear matter has been collected. A full theoretical description of hadronization in nuclei in one consistent framework, including partonic and hadronic (absorption plus rescattering) mechanisms is badly needed. Clearly it will be a challenge for any theoretical model that is developed to describe these data for the various hadrons and nuclei as a function of all kinematic variables, but if successful, this combination of data and theoretical interpretation will contribute essentially to the understanding of non-perturbative QCD at normal, and thence higher densities.

\section{Acknowledgements}

We gratefully acknowledge the DESY management for its support and the staff at DESY and the collaborating institutions for their significant effort. This work was supported by the FWO-Flanders, Belgium; the Natural Sciences and Engineering Research Council of Canada; the National Natural Science Foundation of China; the Alexander von Humboldt Stiftung; the German Bundesministerium für Bildung und Forschung (BMBF); the Deutsche Forschungsgemeinschaft (DFG); the Italian Istituto Nazionale di Fisica Nucleare (INFN); the Monbusho International Scientific Research Program, JSPS, and Toray Science Foundation of Japan; the Dutch Foundation for Fundamenteel Onderzoek der Materie (FOM); the UK Engineering and Physical Sciences Research Council, the Particle Physics and Astronomy Research Council and the Scottish Universities Physics Alliance; the US Department of Energy (DOE) and the National Science Foundation (NSF); and the Ministry of Trade and Economical Development and the Ministry of Education and Science of Armenia.

\section{Appendix A}

In Table A.1 the average values of the kinematic variables that were integrated over when showing the dependences on $v, z, Q^{2}$ and $p_{t}^{2}$ in Figs. 2, 3, 4 are given for the case of pion production on krypton. These values hardly depend on the target used.

Table A.1

Average values of $v, Q^{2}, z$, and $p_{t}^{2}$ for pions produced on krypton

\begin{tabular}{ccccc}
\hline$v$ range & $\langle v\rangle(\mathrm{GeV})$ & $\langle z\rangle$ & $\left\langle Q^{2}\right\rangle\left(\mathrm{GeV}^{2}\right)$ & $\left\langle p_{t}^{2}\right\rangle\left(\mathrm{GeV}^{2}\right)$ \\
\hline $4.0-6.0$ & 5.269 & 0.583 & 1.889 & 0.245 \\
$6.0-8.0$ & 7.151 & 0.501 & 2.029 & 0.164 \\
$8.0-10.0$ & 9.092 & 0.448 & 2.203 & 0.141 \\
$10.0-12.0$ & 11.030 & 0.421 & 2.352 & 0.147 \\
$12.0-14.0$ & 13.009 & 0.406 & 2.506 & 0.171 \\
$14.0-16.0$ & 14.991 & 0.392 & 2.595 & 0.199 \\
$16.0-18.0$ & 16.980 & 0.370 & 2.584 & 0.234 \\
$18.0-20.0$ & 18.964 & 0.349 & 2.475 & 0.275 \\
$20.0-23.5$ & 21.540 & 0.326 & 2.164 & 0.334
\end{tabular}




\section{RAPID COMMUNICATION}

Table A.1 (continued)

\begin{tabular}{|c|c|c|c|c|}
\hline$v$ range & $\langle v\rangle(\mathrm{GeV})$ & $\langle z\rangle$ & $\left\langle Q^{2}\right\rangle\left(\mathrm{GeV}^{2}\right)$ & $\left\langle p_{t}^{2}\right\rangle\left(\mathrm{GeV}^{2}\right)$ \\
\hline $0.1-0.2$ & 0.155 & 18.358 & 2.463 & 0.104 \\
\hline $0.2-0.3$ & 0.247 & 15.953 & 2.623 & 0.164 \\
\hline $0.3-0.4$ & 0.345 & 14.796 & 2.655 & 0.219 \\
\hline $0.4-0.5$ & 0.446 & 14.315 & 2.635 & 0.260 \\
\hline $0.5-0.6$ & 0.546 & 13.963 & 2.585 & 0.284 \\
\hline $0.6-0.7$ & 0.646 & 13.637 & 2.500 & 0.286 \\
\hline $0.7-0.8$ & 0.746 & 12.824 & 2.392 & 0.246 \\
\hline $0.8-0.9$ & 0.845 & 11.841 & 2.252 & 0.192 \\
\hline $0.9-1.3$ & 0.952 & 10.742 & 2.258 & 0.156 \\
\hline$Q^{2}$ range & $\left\langle Q^{2}\right\rangle\left(\mathrm{GeV}^{2}\right)$ & $\langle v\rangle(\mathrm{GeV})$ & $\langle z\rangle$ & $\left\langle p_{t}^{2}\right\rangle\left(\mathrm{GeV}^{2}\right)$ \\
\hline $1.0-1.5$ & 1.360 & 14.790 & 0.399 & 0.217 \\
\hline $1.5-2.0$ & 1.911 & 14.646 & 0.395 & 0.212 \\
\hline $2.0-3.0$ & 2.667 & 14.874 & 0.389 & 0.213 \\
\hline $3.0-4.0$ & 3.746 & 15.062 & 0.382 & 0.213 \\
\hline $4.0-5.0$ & 4.775 & 15.100 & 0.380 & 0.211 \\
\hline $5.0-6.0$ & 5.798 & 15.065 & 0.380 & 0.208 \\
\hline $6.0-8.0$ & 7.161 & 14.819 & 0.380 & 0.203 \\
\hline $8.0-25.0$ & 9.735 & 14.583 & 0.386 & 0.192 \\
\hline$p_{t}^{2}$ range & $\left\langle p_{t}^{2}\right\rangle\left(\mathrm{GeV}^{2}\right)$ & $\langle v\rangle(\mathrm{GeV})$ & $\langle z\rangle$ & $\left\langle Q^{2}\right\rangle\left(\mathrm{GeV}^{2}\right)$ \\
\hline $0.00-0.05$ & 0.023 & 13.059 & 0.379 & 2.550 \\
\hline $0.05-0.10$ & 0.073 & 14.107 & 0.390 & 2.611 \\
\hline $0.10-0.30$ & 0.182 & 15.276 & 0.385 & 2.599 \\
\hline $0.30-0.50$ & 0.384 & 16.172 & 0.392 & 2.616 \\
\hline $0.50-0.70$ & 0.585 & 16.669 & 0.424 & 2.635 \\
\hline $0.70-0.90$ & 0.787 & 16.912 & 0.456 & 2.627 \\
\hline $0.90-1.10$ & 0.987 & 17.211 & 0.483 & 2.620 \\
\hline $1.10-1.40$ & 1.225 & 17.471 & 0.505 & 2.648 \\
\hline $1.40-1.95$ & 1.606 & 17.788 & 0.535 & 2.615 \\
\hline $1.95-5.00$ & 2.418 & 18.487 & 0.571 & 2.625 \\
\hline
\end{tabular}

\section{References}

[1] L. Osborne, et al., Phys. Rev. Lett. 40 (1978) 1624.

[2] J. Ashman, et al., EMC Collaboration, Z. Phys. C 52 (1991) 1.

[3] M. Adams, et al., E665 Collaboration, Phys. Rev. D 50 (1994) 1836.

[4] A. Airapetian, et al., HERMES Collaboration, Eur. Phys. J. C 20 (2001) 479.

[5] A. Airapetian, et al., HERMES Collaboration, Phys. Lett. B 577 (2003) 37.

[6] K. Adcox, et al., PHENIX Collaboration, Phys. Rev. Lett. 88 (2002) 242301.

[7] J. Adams, et al., STAR Collaboration, Phys. Rev. Lett. 92 (2004) 112301.

[8] F.W. Bopp, Z. Phys. C 3 (1979) 171;

F.W. Bopp, Z. Phys. C 5 (1980) 155;

T.D. Gottschalk, D.A. Morris, Nucl. Phys. B 288 (1987) 729;

S.J. Brodsky, B.-Q. Ma, Phys. Lett. B 392 (1997) 452;

P. Abreu, et al., DELPHI Collaboration, Phys. Lett. B 479 (2000) 118;

P. Abreu, et al., DELPHI Collaboration, Phys. Lett. B 492 (2000) 398, Erratum.

[9] W.K. Brooks, Fizika B 13 (2004) 321, nucl-ex/0310032.

[10] K. Hafidi, AIP Conf. Proc. 870 (2006) 669-672, nucl-ex/0609005.

[11] See, e.g., P. Jain, B. Pire, J.P. Ralston, Phys. Rep. 271 (1996) 67.

[12] B. Andersson, et al., Phys. Rep. 97 (1983) 31;

B. Andersson, The Lund Model, Cambridge Univ. Press, 1998.

[13] A. Bialas, M. Gyulassy, Nucl. Phys. B 291 (1987) 793. 
[14] B. Kopeliovich, Phys. Lett. B 243 (1990) 141.

[15] A. Bialas, Acta Phys. Pol. B 11 (1980) 475.

[16] M. Gyulassy, M. Plumer, Nucl. Phys. B 346 (1990) 1.

[17] J. Czyzewski, P. Sawicki, Z. Phys. C 56 (1992) 493.

[18] N. Akopov, G. Elbakian, L. Grigoryan, hep-ph/0205123;

N. Akopov, L. Grigoryan, Z. Akopov, Eur. Phys. J. C 44 (2005) 219.

[19] B.G. Zakharov, Pis'ma Zh. Eksp. Teor. Fiz. 63 (1996) 906.

[20] X.F. Guo, X.-N. Wang, Phys. Rev. Lett. 85 (2000) 3591.

[21] E. Wang, X.-N. Wang, Phys. Rev. Lett. 89 (2002) 162301.

[22] F. Arleo, JHEP 0211 (2002) 44;

F. Arleo, Eur. Phys. J. C 30 (2003) 213.

[23] L. Landau, I. Pomeranchuk, Dokl. Akad. Nauk SSSR 92 (1953) 535; A. Migdal, Phys. Rev. 103 (1956) 1811.

[24] R. Baier, Y.L. Dokshitzer, A.H. Mueller, S. Peigne, D. Schiff, Nucl. Phys. B 484 (1997) 265;

R. Baier, Y.L. Dokshitzer, A.H. Mueller, S. Peigne, D. Schiff, Nucl. Phys. B 483 (1997) 291.

[25] A. Accardi, V. Muccifora, H.J. Pirner, Nucl. Phys. A 720 (2003) 131; A. Accardi, et al., Nucl. Phys. A 761 (2005) 67.

[26] B.Z. Kopeliovich, J. Nemchik, E. Predazzi, A. Hayashigaki, Nucl. Phys. A 740 (2004) 211.

[27] T. Falter, U. Mosel, Fizika B 13 (2004) 165;

T. Falter, W. Cassing, K. Gallmeister, U. Mosel, Phys. Lett. B 594 (2004) 61;

T. Falter, W. Cassing, K. Gallmeister, U. Mosel, Phys. Rev. C 70 (2004) 054609.

[28] K. Ackerstaff, et al., HERMES Collaboration, Nucl. Instrum. Methods A 417 (1998) 230.

[29] P. van der Nat, Master's thesis, Vrije Universiteit, Amsterdam, 2002;

P.B. van der Nat, Acta Phys. Pol. B 35 (2004) 139.

[30] L. Lagamba, Ph.D. thesis, XVII ciclo, Università di Bari, Italy, 2005 (in Italian).

[31] T. Shin, Ph.D. thesis, Hampton University, Massachusetts Institute of Technology, 2000.

[32] T. Benisch, et al., Nucl. Instrum. Methods A 471 (2001) 314.

[33] A. Airapetian, et al., HERMES Collaboration, Phys. Rev. D 71 (2005) 012003.

[34] N. Akopov, et al., Nucl. Instrum. Methods A 479 (2002) 511.

[35] B. Hommez, Ph.D. thesis, University of Gent, 2003.

[36] H. Avakian, et al., Nucl. Instrum. Methods A 417 (1998) 69.

[37] A.A. Akhundov, D.Yu. Bardin, N.M. Shumeiko, Sov. J. Nucl. Phys. 26 (1977) 660;

D.Yu. Bardin, N.M. Shumeiko, Sov. J. Nucl. Phys. 29 (1979) 499;

A.A. Akhundov, et al., Sov. J. Nucl. Phys. 44 (1986) 988.

[38] I. Akushevich, N. Shumeiko, A. Soroko, Eur. Phys. J. C 10 (1999) 681.

[39] A. Airapetian, et al., HERMES Collaboration, Phys. Rev. Lett. 90 (2003) 052501.

[40] Durham HEP database, http://durpdg.dur.ac.uk.

[41] J.W. Cronin, et al., Phys. Rev. D 11 (1975) 3105.

[42] A. Accardi, nucl-th/0604041;

A. Accardi, Eur. Phys. J. C 49 (2007) 347, nucl-th/0609010.

[43] H.P. Blok, L. Lapikás, Phys. Rev. C 73 (2006) 038201.

[44] Z. Akopov, Ph.D. thesis, Yerevan Physics Institute, Yerevan, 2006. 